Sharif University of Technology
Scientia Iranica
Transactions E: Industrial Engineering
hCIENTIA

\title{
A novel improved class of ratio-product type exponential estimators of population variance
}

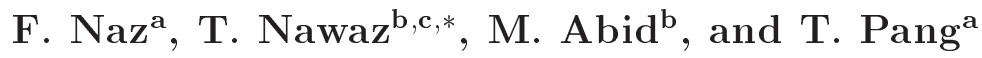 \\ a. School of Mathematical Sciences, Institute of Statistics, Zhejiang University, Hangzhou 310058, China. \\ b. Department of Statistics, Faculty of Physical Sciences, Government College University Faisalabad, Allama Iqbal Road, Faisalabad \\ 38000 , Pakistan. \\ c. School of Mathematical Sciences, Shanghai Jiao Tong University, Minhang Campus, 800 Dongchuan Road, Shanghai 200240, \\ China.
}

Received 4 May 2019; received in revised form 29 July 2020; accepted 21 September 2020

\section{KEYWORDS \\ Auxiliary variable; Mean square error; Percentage relative efficiency; \\ Relative root mean square error; \\ Simple random sampling.}

\begin{abstract}
Several auxiliary information-based estimators of population variance are available in the existing literature on survey sampling. Mostly, these estimators are based on conventional dispersion measures of the auxiliary variable. In this study, a generalized class of ratio-product type exponential estimators of the population variance is proposed by integrating the nonconventional auxiliary information under Simple Random Sampling (SRS). The performance of the proposed estimators was compared, theoretically and numerically, with several existing estimators of the population variance. It was established that the proposed class of estimators outperformed the existing estimators in terms of Mean Squared Error (MSE) and Relative Root Mean Square Error (RRMSE). Moreover, Percentage Relative Efficiency (PRE) of the proposed estimators was much higher than that of their counterparts.
\end{abstract}

(C) 2022 Sharif University of Technology. All rights reserved.

\section{Introduction}

In survey sampling, auxiliary information, if available or easily obtainable without involving much cost, can be advantageously used in choosing the appropriate sampling design, selection of sampling units for inquiry or measurement processes, and the estimation of the characteristics of interest. For example, to study sugar cane production, the auxiliary information about

\footnotetext{
* Corresponding author. Tel.: +86-19802103352; +92-3324248719

E-mail addresses: naz_farah25@zju.edu.cn (F.Naz); tahir.nawaz@gcuf.edu.pk, tahir.nawaz@sjtu.edu.cn (T. Nawaz); mabid@gcuf.edu.pk (M.Abid); txpang@zju.edu.cn (T. Pang)
}

doi: $10.24200 /$ sci. 2020.53481 .3260 area under cultivation, the market price of sugar, the incentive in terms of support price given to the farmers, the production of sugar cane in the previous year, etc. can play a vital role in the efficient estimation of the expected sugar cane production. Ratio, product, regression, and exponential type estimators are popular choices, in practice, to efficiently estimate population mean and variance in the presence of auxiliary information correlated with the study variable. The use of these estimators is expanding to a variety of fields such as yield estimation in agriculture, demographic studies, environmental studies, statistical process monitoring in industry, medical and biological sciences, and many other related fields; see for example [1-7].

Along with population mean, the estimation of variance is of great interest to make certain policy decisions in many practical situations such as agricul- 
ture, business, stock investment, production planning in manufacturing industry, services industry, ecology, seismology, and medical sciences [8-10]. Therefore, efficient estimation of the mean and variance are equally important for effective decision making. The estimation of variance in the context of ratio-type methods of estimation, using auxiliary information, has been considered by various researchers. Usually, conventional auxiliary measures such as mean, median, quartiles, variance, coefficient of kurtosis, variation, skewness, and the correlation between the study and auxiliary variables are employed under ratio and regression type estimation structures to improve the efficiency of the estimators of variance. For example, see [10-27] as well as their cited references for details on this subject. The auxiliary measures used in most of the existing ratio-type estimators of variance are nonresistant to the presence of outliers. The use of such measures can undermine the efficiency of the ratio-type estimators of variance if some outliers are present in the data. Thus, there is need for incorporation of some outlier resistant auxiliary measures to develop more stable ratio-type estimators.

Recently, ratio-type estimators for the estimation of population mean have been developed, which incorporate auxiliary information by using nonconventional measures of location [28-32]. These non-conventional measures are somewhat robust and outlier-resistant, which aids in stabilizing the Mean Square Error (MSE) of estimators in the presence of outliers [8,33,34]. Use of non-conventional or robust measures for the estimation of population variance, in the context of ratio or regression methods of estimation, is still a neglected area. Efficient estimation of variance in the presence of outliers is of paramount interest in several practical situations, such as in agriculture, business, production processes, and so forth. Therefore, the problem of estimating the finite population variance is dealt with in this study by incorporating auxiliary information in some non-conventional and robust measures of dispersion, as detailed in Section 3, to develop more stable and outlier-resistant ratio-product type exponential estimators. It is assumed that the auxiliary information on the non-conventional measures is readily available or can be obtained economically. Suppose a finite population $\Omega=\left\{\Omega_{1}, \Omega_{2}, \cdots, \Omega_{N}\right\}$ consists of $N$ different and identifiable units. Let $(y, x)$ be the measurable study and auxiliary variables, respectively, with their values $\left(y_{i}, x_{i}\right)$ being ascertained on $\Omega_{i}(i=$ $1,2, \cdots, N)$. The purpose of the measurement process is to efficiently estimate the population variance of the variable of interest, $S_{y}^{2}=(N-1)^{-1} \sum_{i=1}^{N}\left(y_{i}-\bar{Y}\right)^{2}$, by drawing a random sample of size $n$ from $\Omega$ using Simple Random Sampling Without Replacement (SRSWOR). Let $s_{y}^{2}=(n-1)^{-1} \sum_{i=1}^{n}\left(y_{i}-\bar{y}\right)^{2}$ and $s_{x}^{2}=(n-$ $1)^{-1} \sum_{i=1}^{n}\left(x_{i}-\bar{x}\right)^{2}$ be the sample variance of the study and the auxiliary variable, respectively. Furthermore, let $\rho_{y x}$ be the population coefficient of correlation between the study and the auxiliary variables and $C_{y}=$ $S_{y} / \bar{Y}$ and $C_{x}=S_{x} / \bar{X}$ be the population coefficients of variation for $y$ and $x$, respectively.

To determine the bias and MSE of the existing and the proposed estimators, the following preliminaries regarding the relative error terms are considered: Let:

$$
\xi_{0}=\left(s_{y}^{2}-S_{y}^{2}\right) / S_{y}^{2},
$$

and:

$$
\xi_{1}=\left(s_{x}^{2}-S_{x}^{2}\right) / S_{x}^{2},
$$

so that:

$$
\begin{aligned}
& E\left(\xi_{1}\right)=E\left(\xi_{2}\right)=0, \\
& E\left(\xi_{0}^{2}\right)=\eta\left(\beta_{2(y)}-1\right)=\beta_{2(y)}^{\star}, \\
& E\left(\xi_{1}^{2}\right)=\eta\left(\beta_{2(x)}-1\right)=\beta_{2(x)}^{\star}, \\
& E\left(\xi_{0} \xi_{1}\right)=\eta\left(\lambda_{22}-1\right)=\lambda_{22}^{\star},
\end{aligned}
$$

where:

$$
\eta=\left(\frac{1}{n}-\frac{1}{N}\right)
$$

$\beta_{2(y)}$ and $\beta_{2(x)}$ are the population coefficients of kurtosis of the study variable $y$ and auxiliary variable $x$, respectively, and:

$$
\lambda_{22}=\frac{\mu_{22}}{\mu_{20} \mu_{02}}
$$

with:

$$
\mu_{r s}=\frac{1}{N-1} \sum_{i=1}^{N}\left(y_{i}-\bar{Y}\right)^{r}\left(x_{i}-\bar{X}\right)^{s} .
$$

\section{Some existing estimators of variance under SRS}

Numerous estimators of finite population variance are available in the literature. In this section, we briefly describe the structure of some of the existing estimators of finite population variance based on SRS.

The usual unbiased estimator of variance under SRS as defined in Cochran [35] is given as:

$$
\widehat{S}_{y}^{2}=\frac{1}{n-1} \sum_{i=1}^{n}\left(y_{i}-\bar{y}\right)^{2} .
$$

The variance of $\widehat{S}_{y}^{2}$ is given as: 


$$
\operatorname{Var}\left(\widehat{S}_{y}^{2}\right) \cong S_{y}^{4} \beta_{2(y)}^{\star}
$$

Isaki [13] proposed a ratio type estimator of $S_{y}^{2}$, which is given as:

$$
\widehat{S}_{R}^{2}=s_{y}^{2}\left(\frac{S_{x}^{2}}{s_{x}^{2}}\right) .
$$

The MSE of $\widehat{S}_{R}^{2}$, to the first degree of approximation, is given as:

$$
\operatorname{MSE}\left(\widehat{S}_{R}^{2}\right) \cong S_{y}^{4}\left[\beta_{2(y)}^{\star}+\beta_{2(x)}^{\star}-2 \lambda_{22}^{\star}\right] .
$$

The conventional regression type estimator, according to Isaki [13], is given as:

$$
\widehat{S}_{R e g}^{2}=s_{y}^{2}+b_{\left(s_{y}^{2}, s_{x}^{2}\right)}\left(S_{x}^{2}-s_{x}^{2}\right)
$$

where $b_{\left(s_{y}^{2}, s_{x}^{2}\right)}$ represents the regression coefficient to be estimated from the sample.

The MSE of $\widehat{S}_{R e g}^{2}$, up to the first degree of approximation, is given as:

$$
\operatorname{MSE}\left(\widehat{S}_{R e g}^{2}\right) \cong S_{y}^{4} \beta_{2(y)}^{\star}\left[1-\rho_{\left(S_{y}^{2}, S_{x}^{2}\right)}^{2}\right]
$$

where:

$$
\rho_{\left(S_{y}^{2}, S_{x}^{2}\right)}=\lambda_{22}^{\star} / \sqrt{\beta_{2(y)}^{\star} \beta_{2(x)}^{\star}},
$$

denotes the population correlation coefficient between $y$ and $x$.

The difference type estimator of Singh et al. [19] is given as:

$$
\widehat{S}_{d}^{2}=c_{1} s_{y}^{2}+c_{2}\left(S_{x}^{2}-s_{x}^{2}\right),
$$

where $c_{1}$ and $c_{2}$ are unknown constants and their optimal values are determined in such a manner that the MSE of $\widehat{S}_{d}^{2}$ is minimized.

The minimum MSE of $\widehat{S}_{d}^{2}$ at optimum values $c_{1(o p t)}=\beta_{2(x)}^{\star} /\left(\beta_{2(x)}^{\star}+\beta_{2(y)}^{\star} \beta_{2(x)}^{\star}-\lambda_{22}^{\star 2}\right)$ and $c_{2(o p t)}=$ $S_{x}^{2} \lambda_{22}^{\star} /\left(S_{y}^{2}\left(\beta_{2(x)}^{\star}+\beta_{2(y)}^{\star} \beta_{2(x)}^{\star}-\lambda_{22}^{\star 2}\right)\right)$, up to the first degree of approximation, is given by:

$$
M S E\left(\widehat{S}_{d}^{2}\right)_{\min } \cong \frac{S_{y}^{4} \beta_{2(y)}^{\star}\left[1-\rho_{\left(S_{y}^{2}, S_{x}^{2}\right)}^{2}\right]}{1+\beta_{2(y)}^{\star}\left[1-\rho_{\left(S_{y}^{2}, S_{x}^{2}\right)}^{2}\right]} .
$$

The ratio-type exponential estimator proposed by Bahl and Tuteja [11] is given as:

$$
\widehat{S}_{B T}^{2}=s_{y}^{2} \exp \left(\frac{S_{x}^{2}-s_{x}^{2}}{S_{x}^{2}+s_{x}^{2}}\right) .
$$

The minimum MSE of $\widehat{S}_{B T}^{2}$, up to the first degree of approximation, is given as:

$$
\operatorname{MSE}\left(\widehat{S}_{B T}^{2}\right) \cong S_{y}^{4}\left[\beta_{2(y)}^{\star}+\frac{1}{4} \beta_{2(x)}^{\star}-\lambda_{22}^{\star}\right] .
$$

Upadhyaya and Singh [25] used the coefficient of kurtosis of the auxiliary variable to propose a modified ratio-type estimator of population variance, which is given as:

$$
\widehat{S}_{U S}^{2}=s_{y}^{2}\left(\frac{S_{x}^{2}+\beta_{2(x)}}{s_{x}^{2}+\beta_{2(x)}}\right) .
$$

The MSE of $\widehat{S}_{U S}^{2}$, up to the first degree of approximation, is given as:

$$
\operatorname{MSE}\left(\widehat{S}_{U S}^{2}\right) \cong S_{y}^{4}\left[\beta_{2(y)}^{\star}+\gamma_{U S}^{2} \beta_{2(x)}^{\star}-2 \gamma_{U S} \lambda_{22}^{\star}\right]
$$

where:

$$
\gamma_{U S}=\frac{S_{x}^{2}}{S_{x}^{2}+\beta_{2(x)}} .
$$

Kadilar and Cingi [14] utilized population coefficient of variation and the population coefficient of kurtosis of the auxiliary variable to suggest some modified estimators of population variance as:

$$
\begin{aligned}
& \widehat{S}_{K C 1}^{2}=s_{y}^{2}\left(\frac{S_{x}^{2}+C_{x}}{s_{x}^{2}+C_{x}}\right), \\
& \widehat{S}_{K C 2}^{2}=s_{y}^{2}\left(\frac{C_{x} S_{x}^{2}+\beta_{2(x)}}{C_{x} s_{x}^{2}+\beta_{2(x)}}\right), \\
& \widehat{S}_{K C 3}^{2}=s_{y}^{2}\left(\frac{\beta_{2(x)} S_{x}^{2}+C_{x}}{\beta_{2(x)} s_{x}^{2}+C_{x}}\right) .
\end{aligned}
$$

The respective MSEs of $\widehat{S}_{K C 1}^{2}, \widehat{S}_{K C 2}^{2}$, and $\widehat{S}_{K C 3}^{2}$, up to the first degree of approximation, are given as:

$$
\begin{aligned}
& \operatorname{MSE}\left(\widehat{S}_{K C 1}^{2}\right) \cong S_{y}^{4}\left[\beta_{2(y)}^{\star}+\gamma_{K C 1}^{2} \beta_{2(x)}^{\star}-2 \gamma_{K C 1} \lambda_{22}^{\star}\right], \\
& M S E\left(\widehat{S}_{K C 2}^{2}\right) \cong S_{y}^{4}\left[\beta_{2(y)}^{\star}+\gamma_{K C 2}^{2} \beta_{2(x)}^{\star}-2 \gamma_{K C 2} \lambda_{22}^{\star}\right], \\
& M S E\left(\widehat{S}_{K C 3}^{2}\right) \cong S_{y}^{4}\left[\beta_{2(y)}^{\star}+\gamma_{K C 3}^{2} \beta_{2(x)}^{\star}-2 \gamma_{K C 3} \lambda_{22}^{\star}\right],
\end{aligned}
$$

where:

$$
\begin{aligned}
\gamma_{K C 1} & =\frac{S_{x}^{2}}{S_{x}^{2}+C_{x}}, \quad \gamma_{K C 2}=\frac{C_{x} S_{x}^{2}}{C_{x} S_{x}^{2}+\beta_{2(x)}}, \\
\gamma_{K C 3} & =\frac{\beta_{2(x)} S_{x}^{2}}{\beta_{2(x)} S_{x}^{2}+C_{x}} .
\end{aligned}
$$

The estimator of population variance $S_{y}^{2}$, given by Shabbir and Gupta [17], is:

$$
\widehat{S}_{S G}^{2}=c_{3} s_{y}^{2}+c_{4}\left(S_{x}^{2}-s_{x}^{2}\right) \exp \left\{\frac{S_{x}^{2}-s_{x}^{2}}{S_{x}^{2}+s_{x}^{2}}\right\},
$$

where $c_{3}$ and $c_{4}$ are unknown quantities to be determined in a manner to minimize the MSE of $\widehat{S}_{S G}^{2}$. 


$$
c_{3(o p t)}=\frac{\beta_{2(x)}^{\star}}{8}\left(\frac{8-\beta_{2(x)}^{\star}}{\beta_{2(x)}^{\star}+\beta_{2(y)}^{\star} \beta_{2(x)}^{\star}-\lambda_{22}^{\star 2}}\right),
$$

and:

$$
c_{4(o p t)}=\frac{S_{y}^{2}}{8 S_{x}^{2}}\left(\frac{-4 \beta_{2(x)}^{\star}+\beta_{2(x)}^{\star}+8 \lambda_{22}^{\star}-\lambda_{22}^{\star} \beta_{2(x)}^{\star}+4 \beta_{2(y)}^{\star} \beta_{2(x)}^{\star}-4 \lambda_{22}^{\star 2}}{\beta_{2(x)}^{\star}+\beta_{2(y)}^{\star} \beta_{2(x)}^{\star}-\lambda_{22}^{\star 2}}\right) .
$$

Box I

The optimum values of $c_{3}$ and $c_{4}$ that minimize the MSE of $\widehat{S}_{S G}^{2}$ are given by the equations shown in Box I, whereas the minimized MSE of $\widehat{S}_{S G}^{2}$ is:

$$
\begin{aligned}
\operatorname{MSE}\left(\widehat{S}_{S G}^{2}\right)_{\min } \cong \frac{S_{y}^{4}}{64} \\
\quad\left(\frac{-\beta_{2(x)}^{\star 2}-16 \beta_{2(y)}^{\star}\left(1-\rho_{\left(S_{y}^{2}, S_{x}^{2}\right)}^{2}\right)\left(\beta_{2(x)}^{\star}-4\right)}{1+\beta_{2(y)}^{\star}\left(1-\rho_{\left(S_{y}^{2}, S_{x}^{2}\right)}^{2}\right)}\right) .
\end{aligned}
$$

Subramani and Kumarapandiyan [36] used the median of the auxiliary variable to propose an estimator of the population variance, which is defined as:

$$
\widehat{S}_{S K 1}^{2}=s_{y}^{2}\left(\frac{S_{x}^{2}+M_{x}}{s_{x}^{2}+M_{x}}\right) .
$$

The MSE of $\widehat{S}_{S K 1}^{2}$, up to the first degree of approximation, is given as:

$$
\operatorname{MSE}\left(\widehat{S}_{S K 1}^{2}\right) \cong S_{y}^{4}\left[\beta_{2(y)}^{\star}+\gamma_{S K 1}^{2} \beta_{2(x)}^{\star}-2 \gamma_{S K 1} \lambda_{22}^{\star}\right],
$$

where:

$$
\gamma_{S K 1}=\frac{S_{x}^{2}}{S_{x}^{2}+M_{x}} .
$$

Taking motivation from Kadilar and Cingi [14] and Subramani and Kumarapandiyan [36], a new ratiotype estimator of the population variance was introduced by Subramani and Kumarapandiyan [22] that utilized the population information for the coefficient of variation and the median of the auxiliary variable, as given below:

$$
\widehat{S}_{S K 2}^{2}=s_{y}^{2}\left(\frac{C_{x} S_{x}^{2}+M_{x}}{C_{x} s_{x}^{2}+M_{x}}\right) .
$$

The MSE of $\widehat{S}_{S K 2}^{2}$, to the first order of approximation, is given as:

$$
\operatorname{MSE}\left(\widehat{S}_{S K 2}^{2}\right) \cong S_{y}^{4}\left[\beta_{2(y)}^{\star}+\gamma_{S K 2}^{2} \beta_{2(x)}^{\star}-2 \gamma_{S K 2} \lambda_{22}^{\star}\right],
$$

where:

$$
\gamma_{S K 2}=\frac{C_{x} S_{x}^{2}}{C_{x} S_{x}^{2}+M_{x}} .
$$

Khan and Shabbir [15] used upper quartile and the population correlation coefficient to suggest an improved ratio estimator of population variance as:

$$
\widehat{S}_{K S}^{2}=s_{y}^{2}\left(\frac{\rho_{y x} S_{x}^{2}+Q_{3(x)}}{\rho_{y x} s_{x}^{2}+Q_{3(x)}}\right) .
$$

The MSE of $\widehat{S}_{K S}^{2}$, up to the first degree of approximation, is given as:

$$
\operatorname{MSE}\left(\widehat{S}_{K S}^{2}\right) \cong S_{y}^{4}\left[\beta_{2(y)}^{\star}+\gamma_{K S}^{2} \beta_{2(x)}^{\star}-2 \gamma_{K S} \lambda_{22}^{\star}\right],
$$

where:

$$
\gamma_{K S}=\frac{\rho_{y x} S_{x}^{2}}{\rho_{y x} S_{x}^{2}+Q_{3(x)}} .
$$

The generalized estimator of population variance proposed by Swain [24] is given below:

$$
\widehat{S}_{S W}^{2}=s_{y}^{2}\left[k\left(\frac{S_{x}^{2}}{s_{x}^{2}}\right)^{q}+(1-k)\left(\frac{s_{x}^{2}}{S_{x}^{2}}\right)^{h}\right]^{\delta},
$$

where $k, q$, and $h$ are suitably chosen constant and $\delta=(1,-1)$. The minimum MSE of $\widehat{S}_{S W}^{2}$, up to the first degree of approximation, at the optimum value of $k=\left(\delta h+\left(\lambda_{22}^{\star} / \beta_{2(y)}^{\star}\right)\right) /(\delta(g+h))$, is given by:

$$
\operatorname{MSE}\left(\widehat{S}_{S W}^{2}\right)_{\min } \cong S_{y}^{4} \beta_{2(y)}^{\star}\left(1-\rho_{\left(S_{y}^{2}, S_{x}^{2}\right)}^{2}\right) .
$$

It is to be noted that the $\operatorname{MSE}\left(\widehat{S}_{S W}^{2}\right)_{\min }$ is equal to $\operatorname{MSE}\left(\widehat{S}_{\text {Reg }}^{2}\right)$.

The general estimator class for population variance proposed by Yadav et al. [26] is given by:

$$
\begin{aligned}
\widehat{S}_{Y G}^{2}= & {\left[c_{5} s_{y}^{2}+c_{6}\left(S_{x}^{2}-s_{x}^{2}\right)\right]\left\{\lambda\left(\frac{a S_{x}^{2}+b}{a s_{x}^{2}+b}\right)\right.} \\
& \left.+(1-\lambda) \exp \left(\frac{a\left(S_{x}^{2}-s_{x}^{2}\right)}{a\left(S_{x}^{2}+s_{x}^{2}\right)+2 b}\right)\right\},
\end{aligned}
$$


where $c_{5}$ and $c_{6}$ are suitably chosen constants that minimize the MSE of $\widehat{S}_{Y G}^{2}$, while $\lambda$ can take the values of 0 or 1 and $a, b$ are the known values of the auxiliary variable parameters. The minimum MSE of $\widehat{S}_{Y G}^{2}$, up to the first degree of approximation, at the optimum values is:

$c_{5(o p t)}=\left(\frac{1-\frac{1}{8} g^{2}\left(1+3 \lambda+4 \lambda^{2}\right) \beta_{2(x)}^{\star}}{1-\frac{1}{4} g^{2} \lambda(1+3 \lambda) \beta_{2(x)}^{\star 2}+\beta_{2(y)}^{\star}\left(1-\rho_{\left(S_{y}^{2}, S_{x}^{2}\right)}^{2}\right)}\right)$,

and:

$c_{6(o p t)}=\frac{S_{y}^{2}}{S_{x}^{2}}\left(\frac{1}{2} g(1+\lambda)+c_{5(o p t)}\left(\frac{\lambda_{22}^{\star}}{\beta_{2(x)}^{\star}}-g(1+\lambda)\right)\right)$,

is given as:

$$
\begin{gathered}
\operatorname{MSE}\left(\widehat{S}_{Y G}^{2}\right)_{\min } \cong S_{y}^{4}\left\{\left(1-\frac{1}{4} g^{2}(1+\lambda)^{2} \beta_{2(x)}^{\star}\right)\right. \\
\left.-\frac{\left(1-\frac{1}{8} g^{2}\left(1+3 \lambda+4 \lambda^{2}\right) \beta_{2(x)}^{\star}\right)^{2}}{1-\frac{1}{4} g^{2} \lambda(1+3 \lambda) \beta_{2(x)}^{\star 2}+\beta_{2(y)}^{\star}\left(1-\rho_{\left(S_{y}^{2}, S_{x}^{2}\right)}^{2}\right)}\right\}
\end{gathered}
$$

where:

$$
g=\frac{a S_{x}^{2}}{\left(a S_{x}^{2}+b\right)}
$$

The minimum MSE of $\widehat{S}_{Y G}^{2}$, up to the first order of approximation at $(\lambda, a, b)=(1,1,0)$, is given below:

$$
\operatorname{MSE}\left(\widehat{S}_{Y G}^{2}\right)_{\min } \cong S_{y}^{4}\left(\frac{S_{y}^{-4} M S E\left(\widehat{S}_{R e g}^{2}\right)\left(1-\beta_{2(x)}^{\star}\right)}{1-\beta_{2(x)}^{\star}+S_{y}^{-4} M S E\left(\widehat{S}_{R e g}^{2}\right)}\right)_{(16)}
$$

Yadav and Kadilar [37] proposed a ratio-product type estimator of population variance, which is given as:

$$
\begin{aligned}
\widehat{S}_{Y K}^{2}= & s_{y}^{2}\left[\alpha_{1}\left\{\frac{\left(1-\beta_{1}\right) s_{x}^{2}+\beta_{1} S_{x}^{2}}{\beta_{1} s_{x}^{2}+\left(1-\beta_{1}\right) S_{x}^{2}}\right\}\right. \\
& \left.+\left(1-\alpha_{1}\right)\left\{\frac{\beta_{1} s_{x}^{2}+\left(1-\beta_{1}\right) S_{x}^{2}}{\left(1-\beta_{1}\right) s_{x}^{2}+\beta_{1} S_{x}^{2}}\right\}\right],
\end{aligned}
$$

where $\alpha_{1}$ and $\beta_{1}$ are constant.

The minimum MSE of $\widehat{S}_{Y K}^{2}$, up to the first degree of approximation, is given below:

$$
\begin{aligned}
& M S E\left(\widehat{S}_{Y K}^{2}\right)_{\min } \cong S_{y}^{4}\left[\left(\beta_{2(y)}^{\star}+\beta_{2(x)}^{\star}-2 \lambda_{22}^{\star}\right)\right. \\
& \quad+16 \alpha_{1} \beta_{1} \beta_{2(x)}^{\star}\left(1-\alpha_{1}-\beta_{1}+\alpha_{1} \beta_{1}\right) \\
& \left.\quad+4 \lambda_{22}^{\star}\left(\alpha_{1}-\beta_{1}\right)^{2}+4 \beta_{2(x)}^{\star}\left(-\alpha_{1}-\beta_{1}+\alpha_{1}^{2}+\beta_{1}^{2}\right)\right]
\end{aligned}
$$

The minimum MSE of $\widehat{S}_{Y K}^{2}$, up to the first degree of approximation at $\left(\alpha_{1(o p t)}, \beta_{1(o p t)}\right)=\left(\frac{1}{2}, \frac{1}{2}\right)$, is:

$$
\operatorname{MSE}\left(\widehat{S}_{Y K}^{2}\right)_{\min } \cong S_{y}^{4} \beta_{2(y)}^{\star} .
$$

And when $\left(\alpha_{1}, \beta_{1}\right)=\left(\left(\left(\beta_{2(x)}^{\star}-2 \lambda_{22}^{\star}\right) / 2 \beta_{2(x)}^{\star}\right), 0\right)$, the minimum MSE of $\widehat{S}_{Y K}^{2}$ is given as:

$$
\operatorname{MSE}\left(\widehat{S}_{Y K}^{2}\right)_{\min } \cong S_{y}^{4} \beta_{2(y)}^{\star}\left(1-\rho_{\left(S_{y}^{2}, S_{x}^{2}\right)}^{2}\right) .
$$

Recently, Yaqub and Shabbir [27] proposed an improved class of estimators for population variance given as:

$$
\begin{aligned}
& \widehat{S}_{Y S}^{2}=s_{y}^{2}\left[c_{7}+c_{8}\left(S_{x}^{2}-s_{x}^{2}\right)\right]\left(\frac{a S_{x}^{2}+b}{a s_{x}^{2}+b}\right) \\
&\left\{\frac{1}{2} \exp \left(\frac{a\left(S_{x}^{2}-s_{x}^{2}\right)}{\left.a S_{x}^{2}+s_{x}^{2}\right)+2 b}\right)\right. \\
&+\left.f r a c 12 \exp \left(\frac{a\left(s_{x}^{2}-S_{x}^{2}\right)}{a\left(s_{x}^{2}+S_{x}^{2}\right)+2 b}\right)\right\},
\end{aligned}
$$

where $c_{7}$ and $c_{8}$ are suitably chosen constants and $a$ and $b$ be the known population parameters of the auxiliary variable. Assuming $a=1$ and $b=0$, the minimum MSE of $\widehat{S}_{Y S}^{2}$, up to the first degree of approximation, based on the optimum values is calculated by Eq. (20) as shown in Box II.

\section{The proposed generalized estimator of variance}

This section presents a generalized ratio product type exponential estimator of population variance, which incorporates information on some outlier resistant nonconventional measures of dispersion of the auxiliary variable. The non-conventional measures are used in a linear combination within the structure of the proposed estimator to make it robust against possible outliers in the data. The non-conventional somewhat robust measures of the auxiliary variable considered in this study include:

(i) The interquartile range: The interquartile range (IQR) is the difference between the upper quartile $\left(Q_{3(x)}\right)$ and lower quartile $\left(Q_{1(x)}\right)$. Symbolically, it is given as:

$$
I Q R_{x}=Q_{3(x)}-Q_{1(x)} .
$$

It is the most known, somewhat, robust measure of dispersion with a breakdown point of $25 \%$.

(ii) The Gini's mean difference estimator: Gini [38] suggested an estimator of dispersion, which is also known as the Gini's mean difference estimator. It is given as: 


$$
c_{7(o p t)}=\frac{\beta_{2(x)}^{\star}}{2}\left(\frac{1+7\left(1-\beta_{2(x)}^{\star}\right)}{\beta_{2(x)}^{\star 2}+4 \beta_{2(x)}^{\star}\left(1-\beta_{2(x)}^{\star}\right)+4 \beta_{2(y)}^{\star} \beta_{2(x)}^{\star}-4 \lambda_{22}^{\star 2}}\right),
$$

and:

$$
c_{8(o p t)}=\frac{S_{y}^{2}}{2 S_{x}^{2}}\left(\frac{\lambda_{22}^{\star}+7 \lambda_{22}^{\star}\left(1-\beta_{2(x)}^{\star}\right)-8 \beta_{2(x)}^{\star}\left(1-\beta_{2(x)}^{\star}\right)+8 \beta_{2(y)}^{\star} \beta_{2(x)}^{\star}-8 \lambda_{22}^{\star 2}}{\beta_{2(x)}^{\star 2}+4 \beta_{2(x)}^{\star}\left(1-\beta_{2(x)}^{\star}\right)+4 \beta_{2(y)}^{\star} \beta_{2(x)}^{\star}-4 \lambda_{22}^{\star 2}}\right),
$$

hence:

$$
\operatorname{MSE}\left(\widehat{S}_{Y S}^{2}\right)_{\min } \cong \frac{S_{y}^{4}}{16}\left[\frac{64\left(1-\beta_{2(x)}^{\star}\right) S_{y}^{-4} M S E\left(\widehat{S}_{R e g}^{2}\right)-\beta_{2(x)}^{\star 2}}{\beta_{2(x)}^{\star}+4\left(1-\beta_{2(x)}^{\star}\right)+4 S_{y}^{-4} M S E\left(\widehat{S}_{R e g}^{2}\right)}\right] .
$$

$$
\operatorname{GIN}_{x}=\frac{4}{N-1} \sum_{i=1}^{N}\left(\frac{2 i-N-1}{2 N}\right) x_{(i)}
$$

where $x_{(i)}$ denotes the $i$ th order statistics. It is robust to outliers and more efficient than the estimators based on range and standard deviation (cf. [39]).

(iii) The Downton's estimator: Like $G_{I N}$, Downton [40] suggested a robust and highly efficient estimator of dispersion. It is defined as:

$$
D O W_{x}=\frac{2 \sqrt{\pi}}{N(N-1)} \sum_{i=1}^{N}\left(i-\frac{N+1}{2}\right) x_{(i)},
$$

where $x_{(i)}$ denotes the $i$ th order statistics. The asymptotic efficiency of $D O W_{x}$ is $97.8 \%$ (cf. [39]).

(iv) The probability weighted moment estimator: Another similar estimator to $\operatorname{GIN}_{x}$ and $D O W_{x}$ is the probability weighted moment estimator given in [41]. It is defined as:

$$
S P W_{x}=\frac{\sqrt{\pi}}{N^{2}} \sum_{i=1}^{N}(2 i-N-1) x_{(i)}
$$

where $x_{(i)}$ denotes the $i$ th order statistics. Its properties are similar to those of $G I N_{x}$ and $D O W_{x}$, as all these three estimators are proportional to each other.

(v) Median absolute deviation from median: Hampel [42] suggested an estimator based on the median of the absolute deviations taken from the median, which is given as:

$$
\begin{aligned}
& M A D M_{x}=m\left[\operatorname{median}\left|x_{i}-\tilde{X}\right|\right] \\
& \text { for } i=1,2, \cdots, N
\end{aligned}
$$

where $m$ is the consistency coefficient and $\tilde{X}$ denotes the median of the observations. $M A D M_{x}$ is robust against outliers with a breakdown point of $50 \%$, but under normality its efficiency is relatively low, i.e., $37 \%$. To make $M A D M_{x}$ a consistent estimator of $\sigma$ under normal distribution, the value of $m$ is set equal to 1.4826 .

(vi) The median of pairwise distances: Shamos [43] and Bickel and Lehmann [44] suggested an estimator of dispersion based on the median of pairwise distances as median $\left\{\left|x_{i}-x_{l}\right| ; i<l\right\}$. Rousseeuw and Croux [45] suggested to pre-multiply it by 1.0483 to achieve consistency under the Gaussian distribution. The resultant estimator can be defined as:

$$
B n_{x}=1.0483\left[\text { median }\left\{\left|x_{i}-x_{l}\right| ; i<l\right\}\right] .
$$

$B n_{x}$ is somewhat robust to outliers with a breakdown point of $29 \%$ and has a relatively high efficiency (about 86\%) under normality.

(vii) The ordered statistic of subranges: Croux and Rousseeuw [46] proposed a class of locationfree robust estimators of dispersion based on ordered statistics of subranges defined as:

$$
S r_{x}^{\alpha}=C_{\alpha}\left\{\left|x_{(i+[\alpha N]+1)}-x_{(i)}\right|_{\left(\left[\frac{N}{2}\right]-\alpha N\right)}\right\},
$$

where $0<\alpha<0.5$ and $x_{(1)} \leq x_{(2)} \leq \cdots \leq$ $x_{(N)}$ are ordered statistics (here, the symbol [.] represents the integer part). The value of $S r_{x}^{\alpha}$ is determined by first, sorting the observations $x_{i}$ and then, calculating the absolute differences $\left|x_{(i+[\alpha N]+1)}-x_{(i)}\right|$ for $i=1,2, \cdots, N-$ $[\alpha N]-1$. From these calculated quantities, the $\left(\left[\frac{N}{2}\right]-\alpha N\right)$ th order statistics yield the desired 
estimator. The constant $C_{\alpha}$ is chosen in such a way that $S r_{x}^{\alpha}$ becomes a consistent estimator for a given value of $\alpha$. In the present study, we use $\alpha=0.25$, which corresponds to $C_{\alpha}=1.4826$ under normality. $S r_{x}^{\alpha}$ has a $50 \%$ breakdown point and it is more efficient than $M A D M_{x}$ for small samples.

(viii) The trimmed mean of median deviations: Rousseeuw and Croux [47] proposed an estimator with a high breakdown point of $50 \%$ and efficiency of $52 \%$ under normality, which is relatively higher than $M A D M_{x}$. It is defined as:

$$
T n_{x}=\frac{1.38}{h} \sum_{k=1}^{h}\left[\operatorname{median}\left\{\left|x_{i}-x_{l}\right|, i \neq l\right\}\right]_{(k)},
$$

where for each $i=1,2, \cdots, N$, we compute the median of $\left|x_{i}-x_{l}\right|, l=1,2,3, \cdots, N$ that yields $N$ values. The average of the first $h$ order statistics gives the desired estimator (here, $h=\left[\frac{N}{2}\right]+1$, which is roughly half the number of observations).

(ix) The 0.25-quantile of pairwise distances: Similar to $B n_{x}$, Rousseeuw and Croux [45] suggested a robust estimator of dispersion based on the 0.25-quantile of pairwise distances between the observations. It is given as:

$$
Q n_{x}=d\left\{\operatorname{median}\left|x_{i}-x_{l}\right| ; i<l\right\}_{(p)},
$$

where:

$$
p=\left(\begin{array}{l}
h \\
2
\end{array}\right) \approx\left(\begin{array}{l}
N \\
2
\end{array}\right) / 4 \quad \text { and } h=\left[\frac{N}{2}\right]+1 .
$$

Hence, the $p$ th order statistic of the $\left(\begin{array}{c}N \\ 2\end{array}\right)$ interpoint distances yields the desired estimator. The value of $d$ is set equal to 2.2219 for $Q n_{x}$ to be a consistent estimator under normality. The estimator $Q n_{x}$ has a $50 \%$ breakdown point and high Gaussian asymptotic efficiency of $82 \%$.

(x) The median of the median of distances: Rousseeuw and Croux [45] suggested another robust estimator, which has a high breakdown point of $50 \%$. it is defined as:

$$
S n_{x}=q\left[\operatorname{median}_{i}\left\{\operatorname{median}_{j}\left|x_{i}-x_{j}\right| ; i \neq j\right\}\right],
$$

where $q$ is the consistency factor with a default value of 1.1926 under the normal population. To compute $S n_{x}$, first, we determine the median of $\left\{\left|x_{i}-x_{j}\right| ; j=1,2, \cdots, N\right\}$ for each $i$, which results in $N$ values. Finally, the median of these $N$ values yields $S n_{x}$.

For more details on these non-conventional measures of dispersion, the readers may see [38-40,43-47] and the references cited therein.

Taking motivation from Shabbir and Gupta [17] and Naz et al. [34], we integrate the above-mentioned non-conventional robust measures of dispersion to design a stable ratio product type exponential estimator of population variance defined as:

$$
\begin{aligned}
& \widehat{S}_{\mathrm{Pr} o p}^{2}=s_{y}^{2}\left\{p_{1}\left(\frac{\varphi S_{x}^{2}+\theta}{\varphi s_{x}^{2}+\theta}\right)^{\frac{\varphi S_{x}^{2}}{\varphi S_{x}^{2}+\theta}}\right. \\
& \left.\quad+p_{2}\left(\frac{\varphi s_{x}^{2}+\theta}{\varphi S_{x}^{2}+\theta}\right)^{\frac{\varphi S_{x}^{2}}{\varphi S_{x}^{2}+\theta}}\right\} \exp \left\{\frac{\varphi\left(S_{x}^{2}-s_{x}^{2}\right)}{\varphi\left(S_{x}^{2}+s_{x}^{2}\right)+2 \theta}\right\}
\end{aligned}
$$

where $p_{1}$ and $p_{2}$ are suitably chosen constants and their values are to be determined later in such a manner that MSE of $\widehat{S}_{\mathrm{Pr} \text { op }}^{2}$ is minimized. $\varphi$ can either be some known real value or function of a known conventional population parameter-such as $\rho_{\left(s_{y}^{2}, s_{x}^{2}\right)}, C_{x}$, or any other value-of the auxiliary variable, whereas $\theta$ can be one of the above-mentioned non-conventional measures.

Setting $\omega=\frac{\varphi S_{x}^{2}}{\varphi S_{x}^{2}+\theta}$ and expressing $\widehat{S}_{\mathrm{Pr} o p}^{2}$ in terms of $\xi$ 's, we have:

$$
\begin{aligned}
\widehat{S}_{\operatorname{Pr} o p}^{2}= & S_{y}^{2}\left(1+\xi_{0}\right)\left[p_{1}\left\{1+\omega \xi_{1}\right\}^{-\omega}+p_{2}\left\{1+\omega \xi_{1}\right\}^{\omega}\right] \\
& \exp \left[-\frac{\omega \xi_{1}}{2}\left(1+\frac{\omega \xi_{1}}{2}\right)^{-1}\right] .
\end{aligned}
$$

For simplification, expanding Eq. (22) and retaining terms only up to the 2 nd order in $\xi$ 's, we have:

$$
\begin{aligned}
& \widehat{S}_{\mathrm{Pr} o p}^{2}-S_{y}^{2} \cong S_{y}^{2}\left[p _ { 1 } \left\{1+\xi_{0}-\left(\omega^{2}+\frac{\omega}{2}\right) \xi_{1}\right.\right. \\
& \left.+\left(\frac{\omega^{4}}{2}+\omega^{3}+\frac{3}{8} \omega^{2}\right) \xi_{1}^{2}-\left(\omega^{2}+\frac{\omega}{2}\right) \xi_{0} \xi_{1}\right\} \\
& +p_{2}\left\{1+\xi_{0}+\left(\omega^{2}-\frac{\omega}{2}\right) \xi_{1}\right. \\
& \left.\left.+\left(\frac{\omega^{4}}{2}-\omega^{3}+\frac{3}{8} \omega^{2}\right) \xi_{1}^{2}+\left(\omega^{2}-\frac{\omega}{2}\right) \xi_{0} \xi_{1}\right\}-1\right]
\end{aligned}
$$

By applying expectation on both sides of Eq. (23), we get the bias of $\widehat{S}_{P R}^{2}$ as follows:

$$
\begin{aligned}
& \operatorname{Bias}\left(\widehat{S}_{P R}^{2}\right) \cong S_{y}^{2}\left[\left(p_{1}+p_{2}-1\right)\right. \\
& +p_{1}\left\{\left(\frac{\omega^{4}}{2}+\omega^{3}+\frac{3}{8} \omega^{2}\right) \beta_{2(x)}^{\star}-\left(\omega^{2}+\frac{\omega}{2}\right) \lambda_{22}^{\star}\right\} \\
& \left.+p_{2}\left\{\left(\frac{\omega^{4}}{2}-\omega^{3}+\frac{3}{8} \omega^{2}\right) \beta_{2(x)}^{\star}+\left(\omega^{2}-\frac{\omega}{2}\right) \lambda_{22}^{\star}\right\}-1\right] .
\end{aligned}
$$




$$
p_{1(o p t)}=\frac{1}{8}\left[\frac{\left\{\begin{array}{l}
\omega(-1+2 \omega)\left(\omega^{2}(-7+4 \omega(1+\omega)) \beta_{2(x)}^{\star 2}-16 \lambda_{22}^{\star 2}+8 \beta_{2(x)}^{\star}\left(1+3 \omega \lambda_{22}^{\star}\right)\right) \\
+16\left(\omega \beta_{2(x)}^{\star}-\lambda_{22}^{\star}\right) \beta_{2(y)}^{\star}+16 \lambda_{22}^{\star}
\end{array}\right\}}{\omega^{2}\left\{\omega^{2}\left(4 \omega^{2}-5\right) \beta_{2(x)}^{\star 2}-16 \lambda_{22}^{\star 2}+4 \beta_{2(x)}^{\star}\left(1+4 \omega \lambda_{22}^{\star}+\beta_{2(y)}^{\star}\right)\right\}}\right],
$$

and:

$$
p_{2(o p t)}=\frac{1}{8}\left[\frac{\left\{\begin{array}{l}
\omega(1+2 \omega)\left(\omega^{2}(-7+4 \omega(-1+\omega)) \beta_{2(x)}^{\star 2}-16 \lambda_{22}^{\star 2}+8 \beta_{2(x)}^{\star}\left(1+3 \omega \lambda_{22}^{\star}\right)\right) \\
+16\left(-\omega \beta_{2(x)}^{\star}+\lambda_{22}^{\star}\right) \beta_{2(y)}^{\star}-16 \lambda_{22}^{\star}
\end{array}\right\}}{\omega^{2}\left\{\omega^{2}\left(4 \omega^{2}-5\right) \beta_{2(x)}^{\star 2}-16 \lambda_{22}^{\star 2}+4 \beta_{2(x)}^{\star}\left(1+4 \omega \lambda_{22}^{\star}+\beta_{2(y)}^{\star}\right)\right\}}\right] .
$$

Box III

$$
M S E\left(\widehat{S}_{P R}^{2}\right)_{\min } \cong \frac{S_{y}^{4}}{16}\left[\frac{\left\{\begin{array}{c}
16 \omega^{2} \beta_{2(x)}^{\star 2}\left(\left(\omega-4 \omega^{3}\right) \lambda_{22}^{\star}-4 \beta_{2(y)}^{\star}\right)-\omega^{4}\left(1-4 \omega^{2}\right)^{2} \beta_{2(x)}^{\star 3}-64 \lambda_{22}^{\star 2}\left(1+\beta_{2(y)}^{\star}\right) \\
+16 \beta_{2(x)}^{\star}\left(\omega^{2}\left(4 \omega^{2}-1\right) \lambda_{22}^{\star 2}+4\left(1+2 \omega \lambda_{22}^{\star}\right) \beta_{2(y)}^{\star}\right)
\end{array}\right.}{\omega^{2}\left(4 \omega^{2}-5\right) \beta_{2(x)}^{\star 2}-16 \lambda_{22}^{\star 2}+4 \beta_{2(x)}^{\star}\left(1+4 \omega \lambda_{22}^{\star}+\beta_{2(y)}^{\star}\right)}\right]
$$

\section{Box IV}

Squaring both sides of Eq. (23) and then, applying expectation, we get the MSE of $\widehat{S}_{P R}^{2}$ as follows:

$$
\begin{aligned}
& M S E\left(\widehat{S}_{P R}^{2}\right)=S_{y}^{4}+p_{1}^{2} S_{y}^{4}\left\{1+\beta_{2(y)}^{\star}\right. \\
& \left.+\left(2 \omega^{4}+3 \omega^{3}+\omega^{2}\right) \beta_{2(x)}^{\star}-4\left(\omega^{2}+\frac{\omega}{2}\right) \lambda_{22}^{\star}\right\} \\
& +p_{2}^{2} S_{y}^{4}\left\{1+\beta_{2(y)}^{\star}+\left(2 \omega^{4}-3 \omega^{3}+\omega^{2}\right) \beta_{2(x)}^{\star}\right. \\
& \left.+4\left(\omega^{2}-\frac{\omega}{2}\right) \lambda_{22}^{\star}\right\}+2 p_{1} p_{2} S_{y}^{4}\left\{1+\beta_{2(y)}^{\star}\right. \\
& \left.+\omega^{2} \beta_{2(x)}^{\star}-2 \omega \lambda_{22}^{\star}\right\}-2 p_{1} S_{y}^{4}\{1
\end{aligned}
$$

$$
\begin{aligned}
& \left.+\left(\frac{\omega^{4}}{2}+\omega^{3}+\frac{3}{8} \omega^{2}\right) \beta_{2(x)}^{\star}-\left(\omega^{2}+\frac{\omega}{2}\right) \lambda_{22}^{\star}\right\} \\
& -2 p_{2} S_{y}^{4}\left\{1+\left(\frac{\omega^{4}}{2}-\omega^{3}+\frac{3}{8} \omega^{2}\right) \beta_{2(x)}^{\star}\right. \\
& \left.+\left(\omega^{2}-\frac{\omega}{2}\right) \lambda_{22}^{\star}\right\} .
\end{aligned}
$$

To get the optimal values of $p_{1}$ and $p_{2}$, we minimize Eq. (25) with respect to $p_{1}$ and $p_{2}$, which gives the equations shown in Box III. The optimal values of $p_{1}$ and $p_{2}$ are then substituted in Eq. (25), which gives the minimum MSE of $\widehat{S}_{P R}^{2}$ by Eq. (26) as shown in Box IV. Many estimators of population variance can be generated from the class of estimators given in Eq. (21) by setting different values of $\varphi$ and $\theta$. A number of selected estimators, which are members of the proposed class, are given in Table 1. 
Table 1. Some new members of the proposed class I estimators.

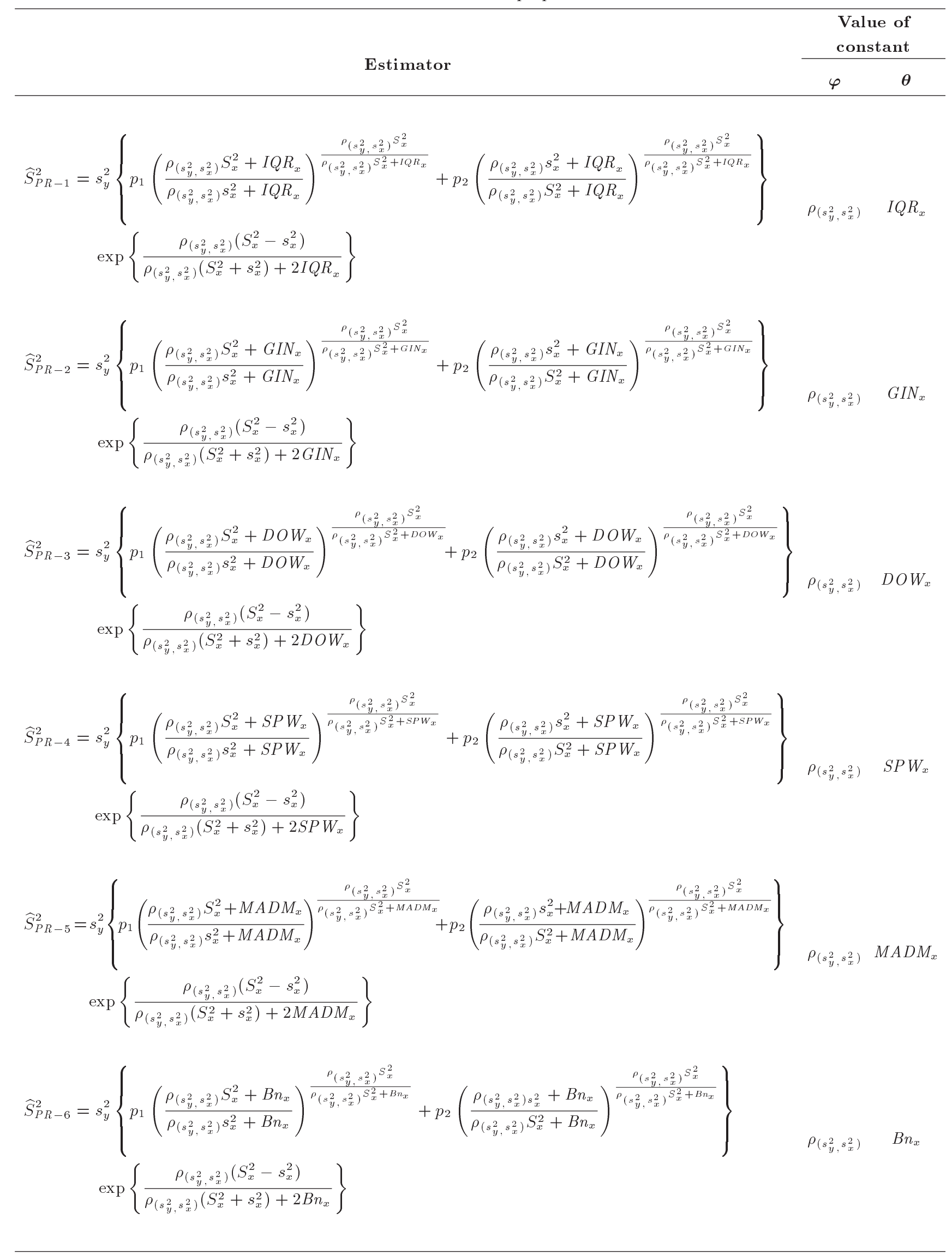


Table 1. Some new members of the proposed class I estimators (continued).

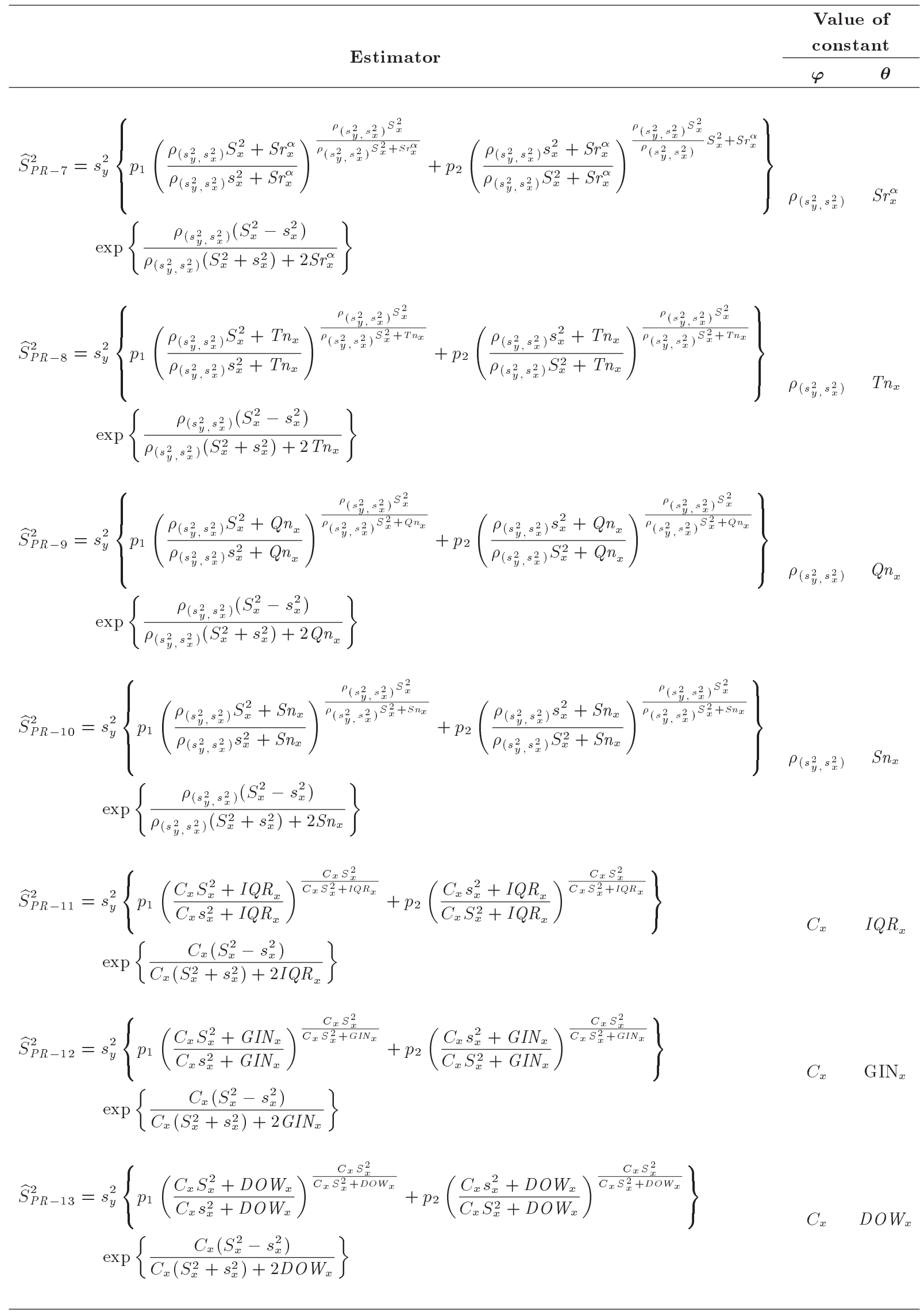


Table 1. Some new members of the proposed class I estimators (continued).

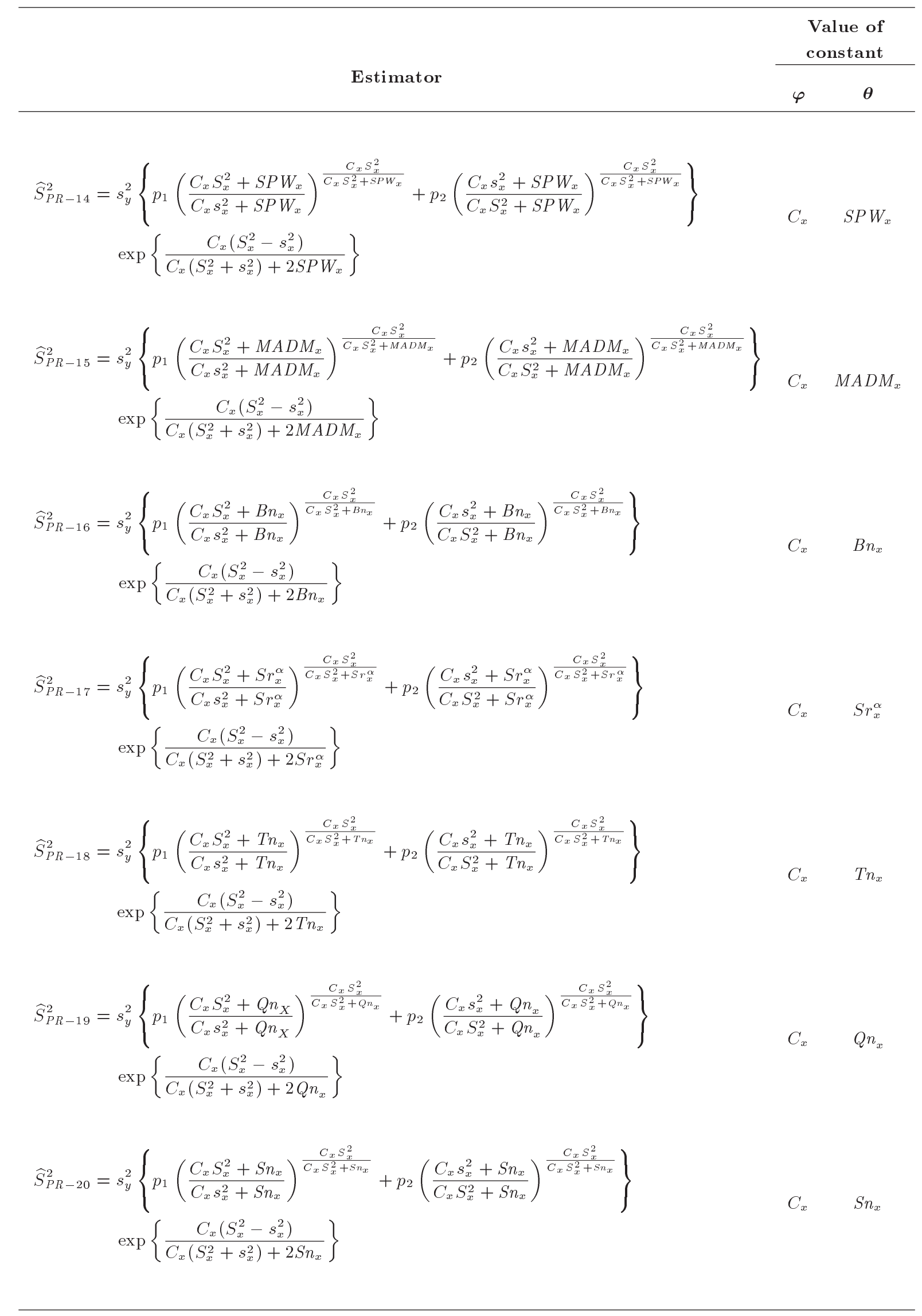




\section{Theoretical and numerical efficiency comparisons}

In this section, theoretical and numerical efficiency of the proposed generalized class of ratio-product type exponential estimators of population variance are compared with the existing estimators discussed in Section 2 .

\subsection{Theoretical comparison}

For theoretical efficiency comparison, let the MSE of the proposed class of estimators $\widehat{S}_{P R}^{2}$ be written as:

$$
M S E\left(\widehat{S}_{P R}^{2}\right)_{\min }=\frac{S_{y}^{4}}{16}\left(\frac{A}{B}\right),
$$

where:

$$
\begin{aligned}
A= & 16 \omega^{2} \beta_{2(x)}^{\star 2}\left(\left(\omega-4 \omega^{3}\right) \lambda_{22}^{\star}-4 \beta_{2(y)}^{\star}\right) \\
& -\omega^{4}\left(1-4 \omega^{2}\right)^{2} \beta_{2(x)}^{\star 3}-64 \lambda_{22}^{\star 2}\left(1+\beta_{2(y)}^{\star}\right) \\
& +16 \beta_{2(x)}^{\star}\left(\omega^{2}\left(4 \omega^{2}-1\right) \lambda_{22}^{\star 2}+4\left(1+2 \omega \lambda_{22}^{\star}\right) \beta_{2(y)}^{\star}\right),
\end{aligned}
$$

and:

$$
\begin{aligned}
B= & \omega^{2}\left(4 \omega^{2}-5\right) \beta_{2(x)}^{\star 2}-16 \lambda_{22}^{\star 2} \\
& +4 \beta_{2(x)}^{\star}\left(1+4 \omega \lambda_{22}^{\star}+\beta_{2(y)}^{\star}\right) .
\end{aligned}
$$

then:

(i) The proposed class of estimators has superior efficiency to $\widehat{S}_{y}^{2}$ if $\operatorname{Var}\left(\widehat{S}_{y}^{2}\right)-M S E\left(\widehat{S}_{P R}^{2}\right)_{\min }>$ 0 . Thus, by Eqs. (1) and (27), the efficiency condition is given as:

$$
\frac{S_{y}^{4}}{16}\left\{\frac{16 B \beta_{2(y)}^{\star}-A}{B}\right\}>0 .
$$

(ii) Similarly, $\widehat{S}_{P R}^{2}$ is more efficient than $\widehat{S}_{R}^{2}$ if $\operatorname{MSE}\left(\widehat{S}_{R}^{2}\right)-\operatorname{MSE}\left(\widehat{S}_{P R}^{2}\right)_{\min }>0$. Thus, by Eqs. (2) and (27), the efficiency condition is given as:

$$
\frac{S_{y}^{4}}{16}\left\{\frac{16 B\left(\beta_{2(y)}^{\star}+\beta_{2(x)}^{\star}-2 \lambda_{22}^{\star}\right)-A}{B}\right\}>0 .
$$

(iii) The estimators envisaged in the class $\widehat{S}_{P R}^{2}$ attain higher efficiency than $\widehat{S}_{R e g}^{2}, \widehat{S}_{S W}^{2}$, and $\widehat{S}_{Y K}^{2}$ if:

$$
\begin{aligned}
& M S E\left(\widehat{S}_{R e g}^{2}\right)-M S E\left(\widehat{S}_{P R}^{2}\right)_{\min }>0, \\
& M S E\left(\widehat{S}_{S W}^{2}\right)_{\min }-M S E\left(\widehat{S}_{P R}^{2}\right)_{\min }>0, \text { and } \\
& M S E\left(\widehat{S}_{Y K}^{2}\right)_{\min }-M S E\left(\widehat{S}_{P R}^{2}\right)_{\min }>0 .
\end{aligned}
$$

Thus, by Eqs. (3), (14), (19), and (27), the efficiency condition is given as:

$$
\frac{S_{y}^{4}}{16}\left\{\frac{16 B \beta_{2(y)}^{\star}\left(1-\rho_{\left(S_{y}^{2}, S_{x}^{2}\right)}^{2}\right)-A}{B}\right\}>0 .
$$

(iv) The efficiency of the proposed class $\widehat{S}_{P R}^{2}$ is higher than $\widehat{S}_{d}^{2}$ if $\operatorname{MSE}\left(\widehat{S}_{d}^{2}\right)_{\min }-\operatorname{MSE}\left(\widehat{S}_{P R}^{2}\right)_{\min }>$ 0 . Thus, by Eqs. (4) and (27), the efficiency condition is given by the equation shown in Box V.

(v) $\widehat{S}_{P R}^{2}$ shows better efficiency than $\widehat{S}_{B T}^{2}$ if $\operatorname{MSE}\left(\widehat{S}_{B T}^{2}\right)-\operatorname{MSE}\left(\widehat{S}_{P R}^{2}\right)_{\min }>0$. Thus, by Eqs. (5) and (27), the efficiency condition is given as:

$$
\frac{S_{y}^{4}}{16}\left\{\frac{4 B\left(4 \beta_{2(y)}^{\star}+\beta_{2(x)}^{\star}-4 \lambda_{22}^{\star}\right)-A}{B}\right\}>0 .
$$

(vi) The proposed class of estimators $\widehat{S}_{P R}^{2}$ display superior efficiency to $\widehat{S}_{U S}^{2}, \widehat{S}_{K C 1}^{2}, \widehat{S}_{K C 2}^{2}, \widehat{S}_{K C 3}^{2}$, $\widehat{S}_{S K 1}^{2}, \widehat{S}_{S K 2}^{2}$, and $\widehat{S}_{K S}^{2}$ if their MSEs are greater than $M S E\left(\widehat{S}_{P R}^{2}\right)_{\min }$. Thus, by Eqs. (6)-(9), (11)-(13), and (27), the efficiency condition is given as:

$$
\frac{S_{y}^{4}}{16}\left\{\frac{16 B\left(\beta_{2(y)}^{\star}+\gamma_{i}^{2} \beta_{2(x)}^{\star}-2 \gamma_{i} \lambda_{22}^{\star}\right)-A}{B}\right\}>0,
$$

where $\gamma_{i}=\gamma_{U S}, \gamma_{K C 1}, \gamma_{K C 2}, \gamma_{K C 3}, \gamma_{S K 1}, \gamma_{S K 2}$, and $\gamma_{K S}$, respectively.

(vii) The efficiency of $\widehat{S}_{P R}^{2}$ is higher than $\widehat{S}_{S G}^{2}$ if $\operatorname{MSE}\left(\widehat{S}_{S G}^{2}\right)_{\min }-\operatorname{MSE}\left(\widehat{S}_{P R}^{2}\right)_{\min }>0$. Thus, by Eqs. (10) and (27), the efficiency condition is given as:

$$
\frac{S_{y}^{4}}{16}\left[\frac{16 B\left\{\beta_{2(y)}^{\star}\left(1-\rho_{\left(S_{y}^{2}, S_{x}^{2}\right)}^{2}\right)\right\}-A\left\{1+\beta_{2(y)}^{\star}\left(1-\rho_{\left(S_{y}^{2}, S_{x}^{2}\right)}^{2}\right)\right\}}{B\left\{1+\beta_{2(y)}^{\star}\left(1-\rho_{\left(S_{y}^{2}, S_{x}^{2}\right)}^{2}\right)\right\}}\right]>0 .
$$




$$
\begin{aligned}
\frac{S_{y}^{4}}{64}\left[\frac{\left\{-\beta_{2(x)}^{\star 2}-16 \beta_{2(y)}^{\star}\left(1-\rho_{\left(S_{y}^{2}, S_{x}^{2}\right)}^{2}\right)\left(\beta_{2(x)}^{\star}-4\right)\right\}}{1+\beta_{2(y)}^{\star}\left(1-\rho_{\left(S_{y}^{2}, S_{x}^{2}\right)}^{2}\right)}\right. \\
\left.\quad-\frac{4 A}{B}\right]>0 .
\end{aligned}
$$

(viii) The estimators envisaged in $\widehat{S}_{P R}^{2}$ achieve higher efficiency than $\widehat{S}_{Y G}^{2}$ if $\operatorname{MSE}\left(\widehat{S}_{Y G}^{2}\right)_{\min }-$ $M S E\left(\widehat{S}_{P R}^{2}\right)_{\min }>0$. Thus, by Eqs. (16) and (27), the efficiency condition is given as:

$$
\begin{aligned}
\frac{S_{y}^{4}}{16}\left[\frac{\left\{16 \beta_{2(y)}^{\star}\left(1-\rho_{\left(S_{y}^{2}, S_{x}^{2}\right)}^{2}\right)\left(1-\beta_{2(x)}^{\star}\right)\right\}}{1-\beta_{2(x)}^{\star}+\beta_{2(y)}^{\star}\left(1-\rho_{\left(S_{y}^{2}, S_{x}^{2} t\right)}^{2}\right)}\right. \\
\left.-\frac{A}{B}\right]>0 .
\end{aligned}
$$

(ix) The proposed estimators of class $\widehat{S}_{P R}^{2}$ are superior to $\widehat{S}_{Y S}^{2}$ in terms of efficiency if $M S E\left(\widehat{S}_{Y S}^{2}\right)_{\min }-\operatorname{MSE}\left(\widehat{S}_{P R}^{2}\right)_{\min }>0$. Thus, by Eqs. (20) and (27), the efficiency condition is given as:

$$
\frac{S_{y}^{4}}{16}\left[\frac{\left\{64 \beta_{2(y)}^{\star}\left(1-\rho_{\left(S_{y}^{2}, S_{x}^{2}\right)}^{2}\right)-\beta_{2(x)}^{\star 2}\right\}}{4-3 \beta_{2(x)}^{\star}+\beta_{2(y)}^{\star}\left(1-\rho_{\left(S_{y}^{2}, S_{x}^{2}\right)}^{2}\right)}-\frac{A}{B}\right]>0 .
$$

\subsection{Numerical comparison in the presence of outliers}

As pointed out in Section 3, the proposed class of estimators incorporate the non-conventional measures, which are somewhat robust to outliers. Therefore, numerical comparison of the proposed class of estimators of population variance with the existing estimators is made by using three population datasets, which contain some outliers. The boxplots given in Figures 13 clearly show that both the study and auxiliary variables in Populations 1 and 3 are crippled with outliers, while population 2 has only one outlier in its auxiliary variable. Thus, these datasets are a good realization of both cases with and without outlier observation. These population datasets are frequently used in many studies to compare the performance of various estimators of population mean and variance (see for example $[28,29,33,48,49]$ ). The description and various population characteristics are detailed as follows:

Population 1: This dataset is taken from Cochran

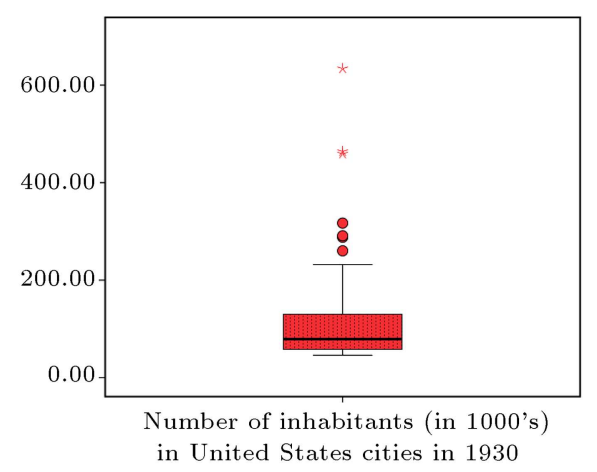

(a)

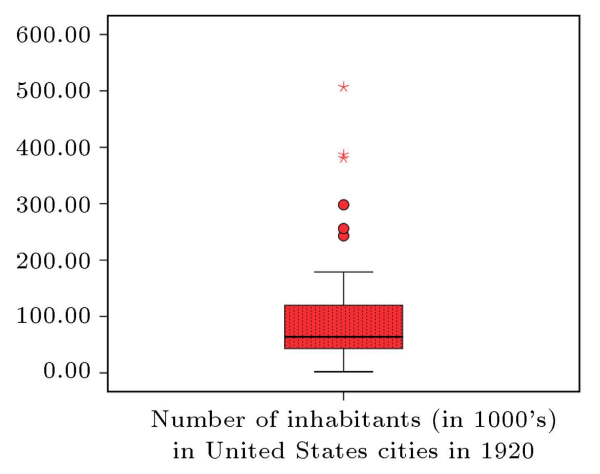

(b)

Figure 1. Boxplots for Population 1. (a) Study variable and (b) auxiliary variable.

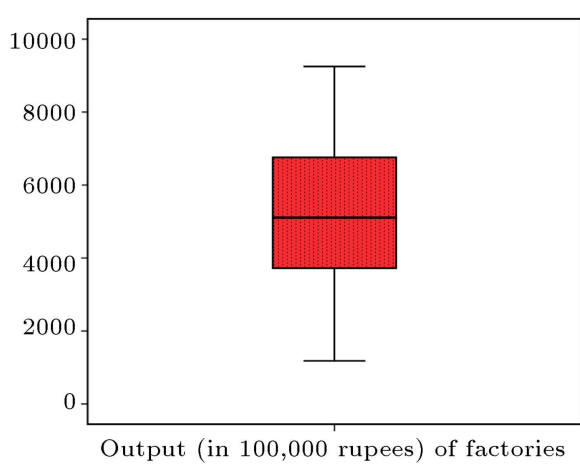

(a)

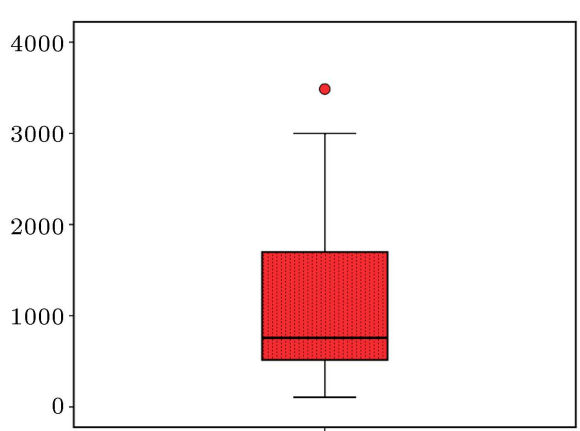

Fixed capital (in 100,000 rupees)

(b)

Figure 2. Boxplots for Population 2. (a) Study variable and (b) auxiliary variable. 


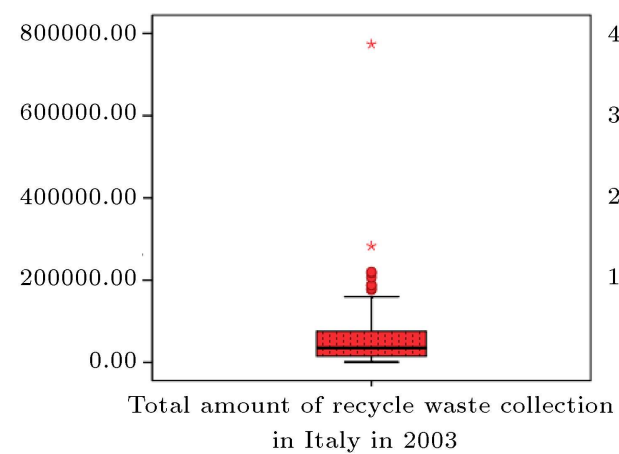

(a)

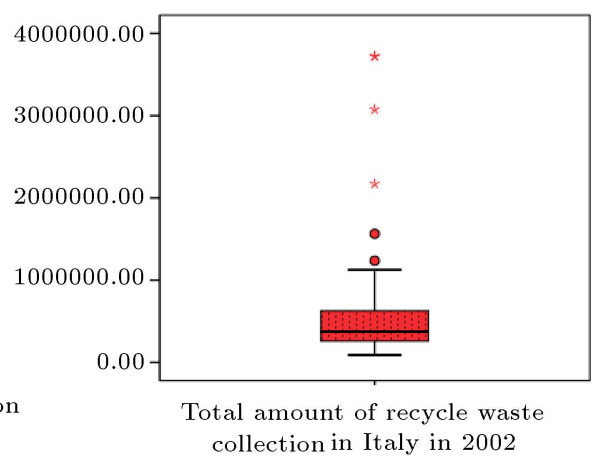

(b)

Figure 3. Boxplots for Population 3: (a) Study variable and (b) auxiliary variable.

[35], where $y$ represents the number of inhabitants (in 1000 's) in United States cities in 1930 and $x$ is the number of inhabitants (in 1000's) in 1920.

$$
\begin{array}{ll}
N=49, \quad n=20, & \bar{Y}=127.7959, \\
\bar{X}=103.1429, & S_{y}^{2}=15158.8299, \\
S_{x}^{2}=10900.4249, & \beta_{2(y)}=4.9245, \\
\beta_{2(x)}=5.9878, & C_{x}=1.0435, \\
\lambda_{22}=4.6977, & \eta=0.02959, \\
\rho_{\left(S_{y}^{2}, S_{x}^{2}\right)}=0.83577, & Q_{1(x)}=43.0, \\
Q_{3(x)}=120.0, & I Q R_{x}=77, \\
G I N_{x}=97.7553, & D O W_{x}=86.6508, \\
S P W_{x}=84.8456, & M A D M_{x}=39.2889, \\
B n_{x}=52.415, & S r_{x}^{\alpha}=34.0998, \\
T n_{x}=35.5488, & Q n_{x}=46.6599, \\
S n_{x}=40.5484, & \rho_{y x}=0.9817, \\
M_{x}=64.0 . &
\end{array}
$$

Population 2: This dataset is obtained from Murthy [50], where $y$ denotes the output (in 100,000 rupees) of factories in a region and $x$ is fixed capital (in 100,000 rupees).

$$
\begin{array}{ll}
N=80, \quad n=20, & \bar{Y}=51.8264, \\
\bar{X}=11.2646, & S_{y}^{2}=336.9757, \\
S_{x}^{2}=70.6634, & \beta_{2(y)}=2.2667, \\
\beta_{2(x)}=2.8664, & C_{x}=0.751,
\end{array}
$$

$$
\begin{array}{ll}
\lambda_{22}=2.2209, & \eta=0.0375, \\
\rho_{\left(S_{y}^{2}, S_{x}^{2}\right)}=0.79311, & Q_{1(x)}=5.1500, \\
Q_{3(x)}=16.975, & I Q R_{x}=11.825, \\
G I N_{x}=10.3613, & D O W_{x}=9.1844, \\
S P W_{x}=9.0681, & M A D M_{x}=4.8925, \\
B n_{x}=7.7060, & S r_{x}^{\alpha}=4.0032, \\
T n_{x}=4.3265, & Q n_{x}=5.1770, \\
S n_{x}=4.6869, & \rho_{y x}=0.941, \\
M_{x}=7.575 . &
\end{array}
$$

Population 3: This dataset is obtained from the Italian Bureau of Environment Protection-IBEP (2004) [source: http://www.osservatorionazionalerifuti.it (20$04)$ ], where $y$ denotes the amount of recyclable waste (in tons) collected in different cities of Italy in 2003 and $x$ is the number of inhabitants living in those cities.

$$
\begin{array}{ll}
N=103, \quad n=40, & \bar{Y}=62.6212, \\
\bar{X}=556.5541, & S_{y}^{2}=8345.7177, \\
S_{y}^{2}=8345.7177, & S_{y}^{2}=8345.7177, \\
\beta_{2(x)}=17.8738, & C_{x}=1.0963, \\
\lambda_{22}=17.2220, & \eta=0.01529, \\
\rho_{\left(S_{y}^{2}, S_{x}^{2}\right)}=0.6570, & Q_{1(x)}=259.3830, \\
Q_{3(x)}=628.0235, & I Q R_{x}=368.6405, \\
G I N_{x}=457.666, & D O W_{x}=405.678, \\
S P W_{x}=401.701, & M A D M_{x}=223.169, \\
B n_{x}=241.697, & S r_{x}^{\alpha}=191.317,
\end{array}
$$


Table 2. Estimated numerical results for the MSEs, RRMSEs, and PREs with respect to $\widehat{S}_{y}^{2}$ of the existing estimators.

\begin{tabular}{|c|c|c|c|c|c|c|c|c|c|}
\hline Estimator & Measure & Pop-1 & Pop-2 & Pop-3 & Estimator & Measure & Pop-1 & Pop-2 & Pop-3 \\
\hline \multirow{3}{*}{$\widehat{S}_{y}^{2}$} & MSE & 26686254 & 5393.75 & 38482180 & \multirow{3}{*}{$\widehat{S}_{S G}^{2}$} & MSE & 7411158 & 1923.464 & 15515489 \\
\hline & RRMSE & 0.3407832 & 0.2179449 & 0.7433034 & & RRMSE & 0.179588 & 0.1301498 & 0.471975 \\
\hline & PRE & 100 & 100 & 100 & & PRE & 360.0821 & 280.4185 & 248.0243 \\
\hline \multirow{3}{*}{$\widehat{S}_{R}^{2}$} & MSE & 10314786 & 2952.368 & 21898276 & \multirow{3}{*}{$\widehat{S}_{S K-1}^{2}$} & MSE & 10213530 & 2494.658 & 21896896 \\
\hline & RRMSE & 0.2118675 & 0.1612452 & 0.5607138 & & RRMSE & 0.2108251 & 0.14822 & 0.5606962 \\
\hline & PRE & 258.7184 & 182.6923 & 175.7316 & & PRE & 261.2834 & 216.212 & 175.7426 \\
\hline \multirow{3}{*}{$\widehat{S}_{R e g}^{2}$} & MSE & 8045752 & 2000.995 & 21871290 & \multirow{3}{*}{$\widehat{S}_{S K-2}^{2}$} & MSE & 10217681 & 2389.708 & 21897016 \\
\hline & RRMSE & 0.1871189 & 0.1327469 & 0.5603682 & & RRMSE & 0.2108679 & 0.1450688 & 0.5606977 \\
\hline & PRE & 331.6813 & 269.5533 & 175.9484 & & PRE & 261.1772 & 225.7074 & 175.7417 \\
\hline \multirow{3}{*}{$\widehat{S}_{d}^{2}$} & MSE & 7773572 & 1966.345 & 16644658 & \multirow{3}{*}{$\widehat{S}_{K S}^{2}$} & MSE & 10124389 & 2162.944 & 21895159 \\
\hline & RRMSE & 0.1839266 & 0.1315925 & 0.4888479 & & RRMSE & 0.209903 & 0.1380143 & 0.5606739 \\
\hline & PRE & 343.2946 & 274.3033 & 231.1984 & & PRE & 263.5839 & 249.3707 & 175.7566 \\
\hline \multirow{3}{*}{$\widehat{S}_{B T}^{2}$} & MSE & 10021370 & 2188.727 & 25695997 & \multirow{3}{*}{$\widehat{S}_{S W}^{2}$} & MSE & 8045752 & 2000.995 & 21871290 \\
\hline & RRMSE & 0.2088324 & 0.1388344 & 0.607392 & & RRMSE & 0.1871189 & 0.1327469 & 0.5603682 \\
\hline & PRE & 266.2935 & 246.4332 & 149.7594 & & PRE & 331.6813 & 269.5533 & 175.9484 \\
\hline \multirow{3}{*}{$\widehat{S}_{U S}^{2}$} & MSE & 10305164 & 2750.183 & 21898209 & \multirow{3}{*}{$\widehat{S}_{Y G}^{2}$} & MSE & 7728302 & 1963.789 & 15367096 \\
\hline & RRMSE & 0.2117687 & 0.155626 & 0.560713 & & RRMSE & 0.1833903 & 0.131507 & 0.4697126 \\
\hline & PRE & 258.96 & 196.1233 & 175.7321 & & PRE & 345.3055 & 274.6603 & 250.4193 \\
\hline \multirow{3}{*}{$\widehat{S}_{K C-1}^{2}$} & MSE & 10313107 & 2895.45 & 21898272 & \multirow{3}{*}{$\widehat{S}_{Y K}^{2}$} & MSE & 8045752 & 2000.995 & 21871290 \\
\hline & RRMSE & 0.2118503 & 0.1596833 & 0.5607138 & & RRMSE & 0.1871189 & 0.1327469 & 0.5603682 \\
\hline & PRE & 258.7606 & 186.2836 & 175.7316 & & PRE & 331.6813 & 269.5533 & 175.9484 \\
\hline \multirow{3}{*}{$\widehat{S}_{K C-2}^{2}$} & MSE & 10305564 & 2691.57 & 21898215 & \multirow{3}{*}{$\widehat{S}_{Y S}^{2}$} & MSE & 7335157 & 1919.253 & 14417414 \\
\hline & RRMSE & 0.2117728 & 0.1539587 & 0.5607131 & & RRMSE & 0.1786648 & 0.1300072 & 0.4549671 \\
\hline & PRE & 258.95 & 200.3942 & 175.732 & & PRE & 363.813 & 281.0338 & 266.9146 \\
\hline \multirow{3}{*}{$\widehat{S}_{K C-3}^{2}$} & MSE & 10314506 & 2932.174 & 21898275 & & & & & \\
\hline & RRMSE & 0.2118647 & 0.1606928 & 0.5607138 & & & & & \\
\hline & PRE & 258.7255 & 183.9505 & 175.7316 & & & & & \\
\hline
\end{tabular}

$$
\begin{array}{ll}
T n_{x}=201.547, & Q n_{x}=223.029, \\
S n_{x}=221.654, & \rho_{y x}=0.7298, \\
M_{x}=373.82 . &
\end{array}
$$

For numerical comparison, we computed the MSEs, Percentage Relative Efficiencies (PREs), and Relative Root Mean Square Errors (RRMSEs) based on the above-mentioned datasets. The PREs of the proposed estimators and the existing estimators relative to the usual SRS estimator of population variance $\left(\widehat{S}_{y}^{2}\right)$ were obtained by using the following expression:

$$
\operatorname{PRE}\left(\bullet, \widehat{S}_{y}^{2}\right)=\frac{\operatorname{Var}\left(\widehat{S}_{y}^{2}\right)}{M S E(\bullet) \operatorname{or} M S E(\bullet)_{\min }} \times 100,
$$

where $M S E(\bullet)$ or $M S E(\bullet)_{\text {min }}$ denotes the MSEs of the existing and proposed estimators of population variance considered in this study. An estimator with a higher value of PRE is considered superior to its counterparts. The RRMSE is obtained by using the following expression:

$$
\begin{aligned}
& R R M S E=\frac{\sqrt{M S E\left(\widehat{\theta}_{i}\right)}}{\theta}, \\
& \widehat{\theta}_{i}=\widehat{S}_{y}^{2}, \widehat{S}_{R}^{2}, \widehat{S}_{R e g}^{2}, \cdots, \widehat{S}_{Y S}^{2}, \widehat{S}_{P R-j}^{2},
\end{aligned}
$$

where $\theta$ is the true population variance, i.e., $S_{y}^{2}$, and $\widehat{S}_{P R-j}^{2}(j=1,2, \cdots, 20)$ denotes the proposed estimators given in Table 1 . An estimator with the lowest RRMSE is usually declared as the most efficient among the competing estimators.

The numerical results for MSEs, RRMSEs, and PREs of the existing estimators and proposed estimators are given in Tables 2 and 3 , respectively. A 
Table 3. Estimated numerical results for the MSEs, RRMSEs, and PREs with respect to $\widehat{S}_{y}^{2}$ of the proposed estimators.

\begin{tabular}{|c|c|c|c|c|c|c|c|c|c|}
\hline Estimator & Measure & Pop-1 & Pop-2 & Pop-3 & Estimator & Measure & Pop-1 & Pop-2 & Pop-3 \\
\hline \multirow{3}{*}{$\widehat{S}_{P R-1}^{2}$} & MSE & 5458119 & 1862.839 & 13835683 & \multirow{3}{*}{$\widehat{S}_{P R-11}^{2}$} & MSE & 5433607 & 1869.284 & 13823937 \\
\hline & RRMSE & 0.1541189 & 0.1280823 & 0.4456938 & & RRMSE & 0.1537724 & 0.1283037 & 0.4455046 \\
\hline & PRE & 488.9277 & 289.5445 & 278.1372 & & PRE & 491.1333 & 288.5463 & 278.3735 \\
\hline \multirow{3}{*}{$\widehat{S}_{P R-2}^{2}$} & MSE & 5490879 & 1847.107 & 13842745 & \multirow{3}{*}{$\widehat{S}_{P R-12}^{2}$} & MSE & 5460177 & 1853.613 & 13828183 \\
\hline & RRMSE & 0.1545807 & 0.1275403 & 0.4458075 & & RRMSE & 0.1541479 & 0.1277647 & 0.445573 \\
\hline & PRE & 486.0106 & 292.0107 & 277.9953 & & PRE & 488.7434 & 290.9857 & 278.288 \\
\hline \multirow{3}{*}{$\widehat{S}_{P R-3}^{2}$} & MSE & 5473413 & 1832.747 & 13838622 & \multirow{3}{*}{$\widehat{S}_{P R-13}^{2}$} & MSE & 5446001 & 1839.234 & 13825704 \\
\hline & RRMSE & 0.1543346 & 0.1270436 & 0.4457412 & & RRMSE & 0.1539477 & 0.1272682 & 0.4455331 \\
\hline & PRE & 487.5615 & 294.2986 & 278.0781 & & PRE & 490.0156 & 293.2607 & 278.3379 \\
\hline \multirow{3}{*}{$\widehat{S}_{P R-4}^{2}$} & MSE & 5470560 & 1831.236 & 13838307 & \multirow{3}{*}{$\widehat{S}_{P R-14}^{2}$} & MSE & 5443688 & 1837.716 & 13825515 \\
\hline & RRMSE & 0.1542944 & 0.1269912 & 0.4457361 & & RRMSE & 0.153915 & 0.1272157 & 0.44553 \\
\hline & PRE & 487.8158 & 294.5414 & 278.0845 & & PRE & 490.2238 & 293.5029 & 278.3418 \\
\hline \multirow{3}{*}{$\widehat{S}_{P R-5}^{2}$} & MSE & 5397326 & 1763.154 & 13824116 & \multirow{3}{*}{$\widehat{S}_{P R-15}^{2}$} & MSE & 5384508 & 1768.603 & 13816990 \\
\hline & RRMSE & 0.1532582 & 0.1246082 & 0.4455075 & & RRMSE & 0.1530761 & 0.1248006 & 0.4453926 \\
\hline & PRE & 494.4348 & 305.9149 & 278.3699 & & PRE & 495.6117 & 304.9723 & 278.5135 \\
\hline \multirow{3}{*}{$\widehat{S}_{P R-6}^{2}$} & MSE & 5418674 & 1812.166 & 13825591 & \multirow{3}{*}{$\widehat{S}_{P R-16}^{2}$} & MSE & 5401720 & 1818.501 & 13817876 \\
\hline & RRMSE & 0.153561 & 0.1263282 & 0.4455312 & & RRMSE & 0.1533205 & 0.1265488 & 0.4454069 \\
\hline & PRE & 492.4868 & 297.6411 & 278.3402 & & PRE & 494.0325 & 296.6042 & 278.4956 \\
\hline \multirow{3}{*}{$\widehat{S}_{P R-7}^{2}$} & MSE & 5388830 & 1744.32 & 13821579 & \multirow{3}{*}{$\widehat{S}_{P R-17}^{2}$} & MSE & 5377668 & 1749.249 & 13815467 \\
\hline & RRMSE & 0.1531375 & 0.1239409 & 0.4454666 & & RRMSE & 0.1529788 & 0.1241158 & 0.4453681 \\
\hline & PRE & 495.2143 & 309.2179 & 278.421 & & PRE & 496.2422 & 308.3467 & 278.5442 \\
\hline \multirow{3}{*}{$\widehat{S}_{P R-8}^{2}$} & MSE & 5391205 & 1751.381 & 13822394 & \multirow{3}{*}{$\widehat{S}_{P R-18}^{2}$} & MSE & 5379580 & 1756.516 & 13815956 \\
\hline & RRMSE & 0.1531713 & 0.1241915 & 0.4454797 & & RRMSE & 0.153006 & 0.1243734 & 0.445376 \\
\hline & PRE & 494.996 & 307.9712 & 278.4046 & & PRE & 496.0658 & 307.0709 & 278.5343 \\
\hline \multirow{3}{*}{$\widehat{S}_{P R-9}^{2}$} & MSE & 5409339 & 1768.802 & 13824105 & \multirow{3}{*}{$\widehat{S}_{P R-19}^{2}$} & MSE & 5394190 & 1774.389 & 13816983 \\
\hline & RRMSE & 0.1534286 & 0.1248076 & 0.4455073 & & RRMSE & 0.1532136 & 0.1250046 & 0.4453925 \\
\hline & PRE & 493.3367 & 304.938 & 278.3701 & & PRE & 494.7222 & 303.9779 & 278.5136 \\
\hline \multirow{3}{*}{$\widehat{S}_{P R-10}^{2}$} & MSE & 5399383 & 1758.961 & 13823996 & \multirow{3}{*}{$\widehat{S}_{P R-20}^{2}$} & MSE & 5386165 & 1764.303 & 13816918 \\
\hline & RRMSE & 0.1532874 & 0.1244599 & 0.4455055 & & RRMSE & 0.1530996 & 0.1246488 & 0.4453915 \\
\hline & PRE & 494.2464 & 306.644 & 278.3723 & & PRE & 495.4592 & 305.7156 & 278.5149 \\
\hline
\end{tabular}

comparison of these results clearly establishes that all the proposed estimators as members of the class $\widehat{S}_{P R}^{2}$ have smaller MSEs and RRMSEs than the existing estimators of population variance in all the populations considered in this study. Moreover, the PREs of the proposed estimators are much higher than those of their existing counterparts. It is also observed that in most cases, the estimators that employ auxiliary information on $C_{x}$ and the non-conventional measures in tandem, that is, $\widehat{S}_{P R-1}^{2}$ to $\widehat{S}_{P R-10}^{2}$, perform slightly better than other estimators of the proposed class $\widehat{S}_{P R}^{2}$.

\section{Conclusion}

In this study, we propose a new generalized class of 
ratio-product type exponential estimators of population variance under Simple Random Sampling (SRS), which incorporates both the conventional and somewhat robust non-conventional auxiliary information. The results for the bias, Mean Square Error (MSE), and efficiency conditions indicated that the proposed estimators were better than the existing estimators in the literature. Using three different datasets, which contained outlier observations, numerical efficiency comparison with the existing estimators was made based on MSEs, Relative Root Mean Square Errors (RRMSEs), and Percentage Relative Efficiency (PREs). It was established that the proposed estimators had superior efficiency to their counterparts.

\section{Acknowledgments}

The authors are thankful to the reviewers and the editor for their valuable comments and suggestions that led to improving the article.

\section{References}

1. Cochran, W.G. "The estimation of the yields of cereal experiments by sampling for the ratio of grain to total produce", The Journal of Agricultural Science, 30(2), pp. 262-275 (1940).

2. Stehman, S.V. "Use of auxiliary data to improve the precision of estimators of thematic map accuracy", Remote Sensing of Environment, 58(2), pp. 169-176 (1996).

3. Ogliore, R.C., Huss, G.R., and Nagashima, K. "Ratio estimation in SIMS analysis", Nuclear Instruments and Methods in Physics Research Section B: Beam Interactions with Materials and Atoms, 269(17), pp. 1910-1918 (2011).

4. Temesgen, H., Monleon, V., Weiskittel, A., et al. "Sampling strategies for efficient estimation of tree foliage biomass", Forest Science, 57(2), pp. 153-163 (2011).

5. Sanusi, R.A., Abujiya, M.R., Riaz, M., et al. "Combined Shewhart CUSUM charts using auxiliary variable", Computers and Industrial Engineering, 105, pp. 329-337 (2017).

6. Raza, M.A., Nawaz, T., and Aslam, M. "On designing CUSUM charts using ratio-type estimators for monitoring the location of normal processes", Scientia Iranica, 27(3), pp. 1593-1605 (2020).

7. Sanusi, R.A., Abbas, N., and Riaz, M. "On efficient CUSUM-type location control charts using auxiliary information", Quality Technology \& Quantitative Management, 15(1), pp. 87-105 (2018).

8. Naz, F., Nawaz, T., Pang, T., et al. "Use of nonconventional dispersion measures to improve the efficiency of ratio-type estimators of variance in the presence of outliers", Symmetry, 12(1), 16 (2020).
9. Özel, G., Çingi, H., and Oğuz, M. "Separate ratio estimators for the population variance in stratified random sampling", Communications in Statistics-Theory and Methods, 43(22), pp. 4766-4779 (2014).

10. Solanki, R.S., Singh, H.P., and Pal, S.K. "Improved ratio-type estimators of finite population variance using quartiles", Hacettepe Journal of Mathematics and Statistics, 44(3), pp. 747-754 (2015).

11. Bahl, S. and Tuteja, R.K. "Ratio and product type exponential estimators", Journal of Information and Optimization Sciences, 12(1), pp. 159-164 (1991).

12. Das, A.K. and Tripathi, T.P. "Use of auxiliary information in estimating the finite population variance", Sankhya C, 40, pp. 139-148 (1978).

13. Isaki, C.T. "Variance estimation using auxiliary information", Journal of the American Statistical Association, 78, pp. 117-123 (1983).

14. Kadilar, C. and Cingi, H. "Ratio estimators for population variance in simple and stratified sampling", $A p$ plied Mathematics and Computation, 173, pp. 10471058 (2006).

15. Khan, M. and Shabbir, J. "A ratio type estimator for the estimation of population variance using quartiles of an auxiliary variable", Journal of Statistics Application and Probability, 2(3), pp. 319-325 (2013).

16. Maqbool, S. and Javaid, S. "Variance estimation using linear combination of tri-mean and quartile average", American Journal of Biological and Environmental Statistics, 3(1), pp. 5-5 (2017).

17. Shabbir, J. and Gupta, S. "On improvement in variance estimation using auxiliary information", Communications in Statistics-Theory and Methods, 36(12), pp. 2177-2185 (2007).

18. Singh, H.P. and Solanki, R.S. "Improved estimation of finite population variance using auxiliary information", Communications in Statistics-Theory and Methods, 42(15), pp. 2718-2730 (2013).

19. Singh, H.P., Upadhyaya, L.N., and Namjoshi, U.D. "Estimation of finite population variance", Current Science, 57(24), pp. 1331-1334 (1988).

20. Singh, R. and Malik, S. "Improved estimation of population variance using information on auxiliary attribute in simple random sampling", Applied Mathematics and Computation, 235, pp. 43-49 (2014).

21. Subramani, J. and Kumarapandiyan, G. "Variance estimation using quartiles and their functions of an auxiliary variable", International Journal of Statistics and Applications, 2(5), pp. 67-72 (2012).

22. Subramani, J. and Kumarapandiyan, G. "Estimation of variance using known coefficient of variation and median of an auxiliary variable", Journal of Modern Applied Statistical Methods, 12(1), pp. 58-64 (2013).

23. Subramani, J. and Kumarapandiyan, G. "A class of modified ratio estimators for estimation of population variance", Journal of Applied Mathematics, Statistics and Informatics, 11(1), pp. 91-114 (2015). 
24. Swain, A.K.P.C. "Generalized estimator of finite population variance", Journal of Statistical Theory and Applications, 14(1), pp. 45-51 (2015).

25. Upadhyaya, L.N. and Singh, H.P. "An estimator for population variance that utilizes the kurtosis of an auxiliary variable in sample surveys", Vikram Mathematical Journal, 19, pp. 14-17 (1999).

26. Yadav, S.K., Kadilar, C., Shabbir, J., et al. "Improved family of estimators of population variance in simple random sampling", Journal of Statistical Theory and Practice, 9(2), pp. 219-226 (2015).

27. Yaqub, M. and Shabbir, J. "An improved class of estimators for finite population variance", Hacettepe Journal of Mathematics and Statistics, 45(5), pp. 1641-1660 (2016).

28. Abid, M., Abbas, N., Nazir, H.Z., et al. "Enhancing the mean ratio estimators for estimating population mean using non-conventional location parameters", Revista Colombiana de Estadística, 39(1), pp. 63-79 (2016).

29. Abid, M., Nazir, H.Z., Riaz, M., et al. "Improved ratio estimators using some robust measures", Hacettepe Journal of Mathematics and Statistics, 47(5), pp. 1375-1393 (2018).

30. Singh, H.P. and Yadav, A. "A new exponential approach for reducing the mean squared errors of the estimators of population mean using conventional and non-conventional location parameters", Journal of Modern Applied Statistical Methods, 18(1), 26 (2020).

31. Subzar, M., Maqbool, S., Raja, T.A., et al. "Improved family of ratio type estimators for estimating population mean using conventional and non conventional location parameters", Revista Investigacion Operacional, 38(5), pp. 499-513 (2017).

32. Abid, M., Sherwani, R.A.K., Abbas, N., et al. "Some improved modified ratio estimators based on decile mean of an auxiliary variable", Pakistan Journal of Statistics and Operation Research, 12(4), pp. 787-797 (2016).

33. Abid, M., Ahmed, S., Tahir, M., et al. "Improved ratio estimators of variance based on robust measures", Scientia Iranica, 26(4), pp. 2484-2494 (2019).

34. Naz, F., Abid, M., Nawaz, T., et al. "Enhancing efficiency of ratio-type estimators of population variance by a combination of information on robust location measures", Scientia Iranica, 27(4), pp. 2040-2056 (2020).

35. Cochran, W.G., Sampling Techniques, 3rd Ed., John Wiley and Sons, New York (1977).

36. Subramani, J. and Kumarapandiyan, G. "Variance estimation using median of the auxiliary variable", International Journal of probability and Statistics, 1(3), pp. 36-40 (2012).

37. Yadav, S.K. and Kadilar, C. "A two parameter variance estimator using auxiliary information", Applied Mathematics and Computation, 226, pp. 117-122 (2014).
38. Gini, C. "Variabilità e mutabilità", in Memorie di Metodologica Statistica Ed., Libreria Eredi Virgilio Veschi, Rome, (1912).

39. David, H.A. "Miscellanea: Gini's mean difference rediscovered", Biometrika, 55(3), pp. 573-575 (1968).

40. Downton, F. "Linear estimates with polynomial coefficients", Biometrika, 53(1-2), pp. 129-141 (1966).

41. Muhammad, F., Ahmad, S., and Abiodullah, M. "Use of probability weighted moments in the analysis of means", Biometrical Journal, 35(3), pp. 371-378 (1993).

42. Hampel, F.R. "The influence curve and its role in robust estimation", Journal of the American Statistical Association, 69(346), pp. 383-393 (1974).

43. Shamos, M.I. "Geometry and Statistics: Problems at the Interface", In New Directions and Recent Results in Algorithms and Complexity, Traub, J.F., Ed., Academic Press, New York, pp. 251-280 (1976).

44. Bickel, P.J. and Lehmann, E.L. "Descriptive statistics for nonparametric models IV. Spread", In Contributions to Statistics, Jureckova, J., Ed., Hajek Memorial Volume, Academia, Prague, pp. 33-40 (1979).

45. Rousseeuw, P.J. and Croux, C. "Alternatives to the median absolute deviation", Journal of the American Statistical Association, 88(424), pp. 1273-1283 (1993).

46. Croux, C. and Rousseeuw, P.J. "A class of highbreakdown scale estimators based on subranges", Communications in Statistics-Theory and Methods, 21(7), pp. 1935-1951 (1992).

47. Rousseeuw, P.J. and Croux, C. "Explicit scale estimators with high breakdown point", In L1-Statistical Analysis and Related Methods, Dodge, Y., Ed., NorthHolland, Amsterdam, pp. 77-92 (1992).

48. Muneer, S., Khalil, A., Shabbir, J., et al. "A new improved ratio-product type exponential estimator of finite population variance using auxiliary information", Journal of Statistical Computation and Simulation, 88(16), pp. 3179-3192 (2018).

49. Singh, H.P., Singh, A.K., and Solanki, R.S. "Estimation of finite population variance using auxiliary information in sample surveys", Statistica, 74(1), pp. 99-116 (2014).

50. Murthy, M.N., Sampling Theory and Methods, Statistical Publishing Society, Calcutta (1967).

\section{Biographies}

Farah Naz earned her MSc and Mphil degrees in Statistics from the Islamia University Bahawalpur, Pakistan, and Government College University Faisalabad, Pakistan, respectively. Currently, she is pursuing $\mathrm{PhD}$ in Statistics in the School of Mathematical Sciences, Institute of Statistics, Zhejiang University, Hangzhou, People's Republic of China, under the Chinese Government Scholarship Program (2017). Her research interest is survey sampling and distribution theory. 
Tahir Nawaz obtained his MSc and MPhil degrees in Statistics from the Islamia University Bahawalpur, Pakistan, and Government College University Lahore, Pakistan, respectively. He served as a statistical officer in Punjab Bureau of Statistics, Pakistan, during May 2007 to November 2009. Also, he served as a lecturer in Statistics at Islamia University Bahawalpur, Pakistan, during November 2009 to October 2013. He has been working as a lecturer in the Department of Statistics, Government College University Faisalabad, Pakistan, since November 2013. He is also pursuing his PhD in Statistics in the School of Mathematical Sciences, Shanghai Jiao Tong University, Shanghai, People's Republic of China, under the Chinese Government Scholarship Program (2016). He has published more than 20 research papers in various research journals. His research interest includes statistical process control, distribution theory, and survey sampling.

Muhammad Abid obtained his MSc and MPhil degrees in Statistics from Quaid-i-Azam University, Islamabad, Pakistan, in 2008 and 2010, respectively.
He received his $\mathrm{PhD}$ in Statistics from the School of Mathematical Sciences, Institute of Statistics, Zhejiang University, Hangzhou, People's Republic of China, in 2017. He served as a statistical officer in National Accounts Wing, Pakistan Bureau of Statistics (PBS), during 2010-2011. He has been serving as an Assistant Professor in the Department of Statistics, Government College University Faisalabad, Pakistan, since 2017. He has published more than 30 research papers in various research journals. His research interests include statistical quality control, Bayesian statistics, nonparametric techniques, and survey sampling.

Tianxiao Pang earned his PhD in Probability and Mathematical Statistics from the Department of Mathematics, Zhejiang University, Hangzhou, People's Republic of China in 2005 and is now an Associate Professor of Statistics at the same university. More than 30 refereed publications in various reputed international journals are to his credit. His research interest is in probability limit theory, large sample theory in statistics, and econometrics. 